\title{
Nucleon resonances with higher spins in soft-wall AdS/QCD
}

\author{
Valery E. Lyubovitskij $\oplus^{1,2,3,4}$ and Ivan Schmidt $\odot^{2}$ \\ ${ }^{1}$ Institut für Theoretische Physik, Universität Tübingen, Kepler Center for Astro and Particle Physics, \\ Auf der Morgenstelle 14, D-72076 Tübingen, Germany \\ ${ }^{2}$ Departamento de Física y Centro Científico Tecnológico de Valparaíso-CCTVal, Universidad Técnica \\ Federico Santa María, Casilla 110-V, Valparaíso, Chile \\ ${ }^{3}$ Department of Physics, Tomsk State University, 634050 Tomsk, Russia \\ ${ }^{4}$ Tomsk Polytechnic University, 634050 Tomsk, Russia
}

(Received 16 September 2020; accepted 7 October 2020; published 11 November 2020)

\begin{abstract}
We present a study of electroexcitation of nucleon resonances with higher spins, in a soft-wall AdS/QCD model, comparing our results with existing data from the CLAS Collaboration at JLab, from MAMI, and other experiments.
\end{abstract}

DOI: 10.1103/PhysRevD.102.094008

\section{INTRODUCTION}

Investigation of electroexcitations of nucleon resonances provides important information about their structure and basic properties [1-8]. For this reason, recent experiments at JLab [4-6] and at MAMI [7,8] aim for a precise determination of the electrocouplings of nucleon resonances and nucleons, supported by theoretical studies of these quantities $[3,8]$. In fact, the strong and electromagnetic structure of nucleon resonances has been studied in detail in many different theoretical approaches, such as in the MAID, SAID, and Bonn-Gatchina partial-wave analysis [8-12], isobar model [13], constituent quark models [14-29], chiral approaches [30-32], light-cone [33] and QCD [34] sum rules, light-front quark models [2,5,35-37], approaches based on solutions of Bethe-Salpeter and Faddeev equations [38-43], approaches used semirelativistic approximation and empirical parametrizations $[44,45]$, and holographic QCD [46-53].

In the past decade significant progress in the study of nucleon resonances has been achieved through the soft-wall AdS/QCD [47-53] formalism. For example, AdS/QCD is able to study the electromagnetic structure of the nucleon and nucleon resonances in the whole region of Euclidean momentum squared $Q^{2}$, and in particular, soft-wall AdS/QCD provides the correct power scaling description of form factors and helicity amplitudes of all hadrons at large $Q^{2}$ [54], while it is also able to give good agreement with the data at low and intermediate $Q^{2}$. In Refs. [47-53] soft-wall AdS/QCD was

Published by the American Physical Society under the terms of the Creative Commons Attribution 4.0 International license. Further distribution of this work must maintain attribution to the author(s) and the published article's title, journal citation, and DOI. Funded by SCOAP ${ }^{3}$. focused on the study of form factors and helicity amplitudes of electroexcitations of the Roper $N(1440)$ (first radial excitation of the nucleon) and the negative-parity state $N^{*}(1535)$. In particular, in Refs. [48,51-53,55] we proposed and developed a soft-wall AdS/QCD framework for the study of nucleon resonances with adjustable quantum numbers and successfully applied it to the unified description of electromagnetic structure of three states - nucleon, Roper, and $N^{*}(1535)$. In the present manuscript we apply this theoretical approach for the study of the electromagnetic structure of nucleon resonances with higher spins.

The paper is organized as follows. In Sec. II we briefly discuss our formalism. In Sec. III we present the analytical calculation and the numerical analysis of electromagnetic form factors and helicity amplitudes of the transitions between nucleon and nucleon resonances. Finally, Sec. IV contains our summary.

\section{FORMALISM}

In this section we discuss the basic principles of our approach [56-62] and focus on its application to nucleon resonances $[48,51-53,56,58]$ with higher spins. First, we define the conformal Poincare metric, which is used in our formalism:

$g_{M N} x^{M} x^{N}=\epsilon_{M}^{a}(z) \epsilon_{N}^{b}(z) \eta_{a b} x^{M} x^{N}=\frac{1}{z^{2}}\left(d x_{\mu} d x^{\mu}-d z^{2}\right)$,

where $\epsilon_{M}^{a}(z)=\delta_{M}^{a} / z$ is the vielbein, $g=\left|\operatorname{det}\left(g_{M N}\right)\right|=1 / z^{10}$.

Next we discuss the construction of the effective action in terms of the 5D AdS fermion fields $\psi_{i, \tau}, \psi_{i, \tau}^{M M_{1} \ldots M_{l-1}}$ and the vector field $V_{M}(x, z)$, where $\tau=N+L$ is the twist defined as the number of partons plus angular orbital momentum. The vector field is dual to the electromagnetic 
field, while the fermion fields are duals to the left- and right-handed chiral doublets of the nucleon and the nucleon resonances, with $\mathcal{O}^{L}=\left(B_{1}^{L}, B_{2}^{L}\right)^{T}$ and $\mathcal{O}^{R}=\left(B_{1}^{R}, B_{2}^{R}\right)^{T}$ where $B_{1}=p, N_{p}^{*}$ and $B_{2}=n, N_{n}^{*}$. These AdS fields are in the fundamental representations of the chiral $S U_{L}(2)$ and $S U_{R}(2)$ subgroups and are holographic analogs of the nucleon $N$ and $N^{*}$ resonance, respectively. They have constrained (confined) dynamics in AdS space, due to the presence of dilaton field $\varphi(z)=\kappa^{2} z^{2}$, where $\kappa$ is its scale parameter. The action $S$ for the description of electroexcitations of nucleon resonances contains a free part $S_{0}$ describing the dynamics of AdS fields, and an interaction part $S_{\text {int }}$ describing the interactions of fermions with the vector field dual to electromagnetic field

$$
\begin{aligned}
S & =S_{0}+S_{\mathrm{int}}, \\
S_{0} & =\int d^{4} x d z \sqrt{g} e^{-\varphi(z)}\left\{\mathcal{L}_{N}(x, z)+\mathcal{L}_{N^{*}}(x, z)+\mathcal{L}_{V}(x, z)\right\}, \\
S_{\mathrm{int}} & =\int d^{4} x d z \sqrt{g} e^{-\varphi(z)} \mathcal{L}_{V N N^{*}}(x, z),
\end{aligned}
$$

where $\mathcal{L}_{N}, \mathcal{L}_{N^{*}}, \mathcal{L}_{V}$, and $\mathcal{L}_{V N N^{*}}$ are the free and interaction Lagrangians, respectively, given by

$$
\begin{aligned}
\mathcal{L}_{N}(x, z) & =\sum_{i=+,-; \tau} c_{\tau} \bar{\psi}_{i, \tau}(x, z) \hat{\mathcal{D}}_{i}(z) \psi_{i, \tau}(x, z), \\
\mathcal{L}_{N^{*}}(x, z) & =\sum_{i=+,-; \tau^{*}} c_{\tau^{*}} \bar{\psi}_{i, \tau^{*}}^{M M_{1} \ldots M_{l-1}}(x, z) \hat{\mathcal{D}}_{i}(z) \psi_{M M_{1} \ldots M_{l-1}, i, \tau^{*}}(x, z), \\
\mathcal{L}_{V}(x, z) & =-\frac{1}{4} V_{M N}(x, z) V^{M N}(x, z), \\
\mathcal{L}_{V N N^{*}}(x, z) & =\sum_{i, M} \sum_{\tau \tau^{*}} g_{\tau \tau^{*}}^{(i M)} J_{\tau \tau^{*}}^{(i M)}(x, z)+\text { H.c. }
\end{aligned}
$$

Here $\tau$ and $\tau^{*}$ are the twists of both the nucleon and nucleon resonance, which run from their minimal possible value. We have introduced the following shortened notations

$$
\begin{aligned}
\hat{\mathcal{D}}_{ \pm}(z) & =\frac{i}{2} \Gamma^{M} \stackrel{\leftrightarrow}{\partial}_{M}-\frac{i}{8} \Gamma^{M} \omega_{M}^{a b}\left[\Gamma_{a}, \Gamma_{b}\right] \mp\left(\mu+U_{F}(z)\right), \\
\hat{\mathcal{V}}_{ \pm, m}^{N^{*} N}(x, z) & =Q \Gamma^{M} V_{M}(x, z)
\end{aligned}
$$

$c_{\tau}, c_{\tau}^{*}, g_{\tau \tau^{*}}^{(i M)}$, and $J_{\tau \tau^{*}}^{(i M)}(x, z)$ are the sets of effective couplings and currents. The currents $J_{\tau \tau^{*}}^{(i M)}(x, z)$ with $i=1,2,3,4$ and $M=A, B, C, D, E, F$ are given, in terms of AdS fermion and vector fields, by

$$
\begin{aligned}
& J_{\tau \tau^{*}}^{(1 M)}(x, z)=J_{++, \tau \tau^{*}}^{(M)}(x, z)+J_{-, \tau \tau^{*}}^{(M)}(x, z), \\
& J_{\tau \tau^{*}}^{(2 M)}(x, z)=J_{++, \tau \tau^{*}}^{(M)}(x, z)-J_{-, \tau \tau^{*}}^{(M)}(x, z), \\
& J_{\tau \tau^{*}}^{(3 M)}(x, z)=J_{+-, \tau \tau^{*}}^{(M)}(x, z)+J_{-+, \tau \tau^{*}}^{(M)}(x, z), \\
& J_{\tau \tau^{*}}^{(4 M)}(x, z)=J_{+-, \tau \tau^{*}}^{(M)}(x, z)-J_{-+, \tau \tau^{*}}^{(M)}(x, z),
\end{aligned}
$$

where

$$
\begin{aligned}
& J_{i j, \tau \tau^{*}}^{(A)}(x, z)=\bar{\psi}_{i, \tau^{*}}^{M M_{1} \ldots M_{l-1}}(x, z) V_{M M_{1} \ldots M_{l-1}}^{(1)}(x, z) \psi_{j, \tau}(x, z), \\
& J_{i j, \tau \tau^{*}}^{(B)}(x, z)=\partial^{K} \bar{\psi}_{i, \tau^{*}}^{M M_{1} \ldots M_{l-1}}(x, z) V_{K M M_{1} \ldots M_{l-1}}^{(2)}(x, z) \psi_{j, \tau}(x, z), \\
& J_{i j, \tau \tau^{*}}^{(C)}(x, z)=\bar{\psi}_{i, \tau^{*}}^{M M_{1} \ldots M_{l-1}}(x, z) V_{M M_{1} \ldots M_{l-1}}^{(3)}(x, z) \psi_{j, \tau}(x, z), \\
& J_{i j, \tau \tau^{*}}^{(D)}(x, z)=\bar{\psi}_{i, \tau^{*}}^{M M_{1} \ldots M_{l-1}}(x, z) i \Gamma^{z} V_{M M_{1} \ldots M_{l-1}}^{(1)}(x, z) \psi_{j, \tau}(x, z), \\
& J_{i j, \tau \tau^{*}}^{(E)}(x, z)=\partial^{K} \bar{\psi}_{i, \tau^{*}}^{M M_{1} \ldots M_{l-1}}(x, z) i \Gamma^{z} V_{K M M_{1} \ldots M_{l-1}}^{(2)}(x, z) \psi_{j, \tau}(x, z), \\
& J_{i j, \tau \tau^{*}}^{(F)}(x, z)=\bar{\psi}_{i, \tau}^{M M_{1} \ldots M_{l-1}}(x, z) i \Gamma^{z} V_{M M_{1} \ldots M_{l-1}}^{(3)}(x, z) \psi_{j, \tau}(x, z),
\end{aligned}
$$

and 


$$
\begin{aligned}
V_{M M_{1} \ldots M_{l-1}}^{(1)}(x, z) & =\partial_{M_{1}} \ldots \partial_{M_{l-1}} i \Gamma^{K} V_{K M}(x, z), \\
V_{K M M_{1} \ldots M_{l-1}}^{(2)}(x, z) & =\partial_{M_{1}} \ldots \partial_{M_{l-1}} V_{K M}(x, z), \\
V_{M M_{1} \ldots M_{l-1}}^{(3)}(x, z) & =\partial_{M_{1}} \ldots \partial_{M_{l-1}} \partial^{K} V_{K M}(x, z) .
\end{aligned}
$$

Here $\mu$ is the five-dimensional mass of the spin- $-\frac{1}{2} \operatorname{AdS}$ fermion with $\mu=3 / 2+L$ ( $L$ is the orbital angular momentum); $U_{F}(z)=\varphi(z)$ is the dilaton potential; $Q=$ $\operatorname{diag}(1,0)$ is the charge matrix corresponding to doublets of nucleon (nucleon resonances); $V_{M N}=\partial_{M} V_{N}-\partial_{N} V_{M}$ is the stress tensor for the vector field; $\omega_{M}^{a b}=\left(\delta_{M}^{a} \delta_{z}^{b}-\right.$ $\left.\delta_{M}^{b} \delta_{z}^{a}\right) / z$ is the spin connection term, while $\Gamma^{M}=\epsilon_{a}^{M} \Gamma^{a}$ and $\Gamma^{a}=\left(\gamma^{\mu},-i \gamma^{5}\right)$ are the Dirac matrices in AdS space, $\left[\Gamma_{a}, \Gamma_{b}\right]=\Gamma_{a} \Gamma_{b}-\Gamma_{b} \Gamma_{a}$.

Next we split 5D AdS fermion fields $\psi_{ \pm, \tau}(x, z)$ and $\psi_{ \pm, \tau}^{M M_{1} \ldots M_{l-1}}(x, z)$ into left- and right-chirality components

$$
\begin{aligned}
\psi(x, z) & =\psi_{L}(x, z)+\psi_{R}(x, z), \\
\psi_{L / R}(x, z) & =\frac{1 \mp \gamma^{5}}{2} \psi(x, z)
\end{aligned}
$$

for the nucleon, and

$$
\begin{aligned}
& \psi^{M M_{1} \ldots M_{l-1}}(x, z)=\psi_{L}^{M M_{1} \ldots M_{l-1}}(x, z)+\psi_{R}^{M M_{1} \ldots M_{l-1}}(x, z), \\
& \psi_{L / R}^{M M_{1} \ldots M_{l-1}}(x, z)=\frac{1 \mp \gamma^{5}}{2} \psi^{M M_{1} \ldots M_{l-1}}(x, z)
\end{aligned}
$$

for the nucleon resonances with higher spins and perform the Kaluza-Klein expansion as

$$
\psi_{ \pm, \tau}(x, z)=\frac{1}{\sqrt{2}} \sum_{n}\left[ \pm \psi_{L n}(x) F_{\tau n}^{L / R}(z)+\psi_{R n}(x) F_{\tau n}^{R / L}(z)\right]
$$

$$
\begin{aligned}
& \psi_{ \pm, \tau}^{M M_{1} \ldots M_{l-1}}(x, z) \\
& =\frac{1}{\sqrt{2}} \sum_{n} \epsilon_{a a_{1} \ldots a_{l-1}}^{M M_{1} \ldots M_{l-1}}(z)\left[ \pm \psi_{L n}^{a a_{1} \ldots a_{l-1}}(x) F_{\tau n}^{L / R}(z)\right. \\
& \left.\quad+\psi_{R n}^{a a_{1} \ldots a_{l-1}}(x) F_{\tau n}^{R / L}(z)\right]
\end{aligned}
$$

where $n$ is the radial quantum number and

$$
\epsilon_{a a_{1} \ldots a_{l-1}}^{M M_{1} \ldots M_{l-1}}(z)=\epsilon_{a}^{M}(z) \epsilon_{a_{1}}^{M_{1}}(z) \ldots \epsilon_{a_{l-1}}^{M_{l-1}}(z)
$$

Here

$$
F_{\tau n}^{L / R}(z)=e^{\kappa^{2} z^{2} / 2} z^{2} f_{\tau n}^{L / R}(z)
$$

are the bulk profiles with twist $\tau$ and radial quantum number $n$, which depend on the holographic variable $z$, where

$$
\begin{aligned}
& f_{\tau n}^{L}(z)=\sqrt{\frac{2 \Gamma(n+1)}{\Gamma(\tau+n)}} \kappa^{\tau} z^{\tau-1 / 2} e^{-\kappa^{2} z^{2} / 2} L_{n}^{\tau-1}\left(\kappa^{2} z^{2}\right), \\
& f_{\tau n}^{R}(z)=\sqrt{\frac{2 \Gamma(n+1)}{\Gamma(\tau-1+n)}} \kappa^{\tau-1} z^{\tau-3 / 2} e^{-\kappa^{2} z^{2} / 2} L_{n}^{\tau-2}\left(\kappa^{2} z^{2}\right),
\end{aligned}
$$

TABLE I. Quantum numbers of the nucleon and nucleon resonances.

\begin{tabular}{ccccc}
\hline \hline Baryon & $J^{P}$ & $L$ & $n$ & Mass $(\mathrm{MeV})[63]$ \\
\hline$N(938)$ & $\frac{1}{2}^{+}$ & 0 & 0 & 938.27 \\
$N(1440)$ & $\frac{1}{2}^{+}$ & 0 & 1 & $1370 \pm 10$ \\
$N(1710)$ & $\frac{1}{2}^{+}$ & 0 & 2 & $1700 \pm 20$ \\
$N(1535)$ & $\frac{1}{2}^{-}$ & 1 & 0 & $1510 \pm 10$ \\
$N(1520)$ & $\frac{3}{2}^{-}$ & 1 & 0 & $1510 \pm 5$ \\
$N(1650)$ & $\frac{1}{2}^{-}$ & 1 & 0 & $1655 \pm 15$ \\
$N(1700)$ & $\frac{3}{2}^{-}$ & 1 & 0 & $1700 \pm 50$ \\
$N(1675)$ & $\frac{5}{2}^{-}$ & 1 & 0 & $1660 \pm 5$ \\
$N(1720)$ & $\frac{3}{2}^{+}$ & 2 & 0 & $1675 \pm 15$ \\
$N^{\prime}(1720)$ & $\frac{3}{2}^{+}$ & 2 & 0 & $1720 \pm 15[64]$ \\
$N(1680)$ & $\frac{5}{2}^{+}$ & 2 & 0 & $1675_{-10}^{+5}$ \\
$\Delta(1232)$ & $\frac{3}{2}^{+}$ & 0 & 0 & $1210 \pm 1$ \\
$\Delta(1620)$ & $\frac{1}{2}^{-}$ & 1 & 0 & $1600 \pm 10$ \\
$\Delta(1700)$ & $\frac{3}{2}^{-}$ & 1 & 0 & $1665 \pm 25$ \\
\hline \hline
\end{tabular}

and $L_{n}^{m}(x)$ are the generalized Laguerre polynomials. The bulk profiles $f_{\tau}^{L / R}(z)$ are normalized as

$$
1=\int_{0}^{\infty} d z\left[f_{\tau n}^{L}(z)\right]^{2}=\int_{0}^{\infty} d z\left[f_{\tau n}^{R}(z)\right]^{2}
$$

The nucleon is identified as the ground state with $n=L=0$, while the nucleon resonance has specific values of $n$ and $L$. In Table I we display the quantum numbers (spin-parity $J^{P}$, angular orbital moment $L$, radial quantum number $n$, mass) of the baryons considered in the present paper. The action describing transitions $\frac{1}{2}^{+} \gamma^{*} \rightarrow \frac{1}{2}^{ \pm}$has been derived and discussed in detail in Refs. [48,51-53]. In Appendix we briefly specify this action.

For the vector field $V_{\mu}(x, z)$ we apply the axial gauge $V_{z}=0$ and perform a Fourier transformation with respect to the Minkowski coordinate

$$
V_{\mu}(x, z)=\int \frac{d^{4} q}{(2 \pi)^{4}} e^{i q x} V_{\mu}(q) V(q, z),
$$

where $V(q, z)$ is the vector bulk to boundary (dual to the $q^{2}$ dependent electromagnetic current) obeying the equation of motion

$$
\partial_{z}\left(\frac{e^{-\varphi(z)}}{z} \partial_{z} V(q, z)\right)+q^{2} \frac{e^{-\varphi(z)}}{z} V(q, z)=0
$$

with solution in terms of gamma $\Gamma(n)$ and Tricomi $U(a, b, z)$ functions

$$
V(q, z)=\Gamma\left(1-\frac{q^{2}}{4 \kappa^{2}}\right) U\left(-\frac{q^{2}}{4 \kappa^{2}}, 0, \kappa^{2} z^{2}\right) .
$$

It was shown in Ref. [65] that in the Euclidean region $\left(Q^{2}=-q^{2}>0\right)$ it is convenient to use the integral representation for $V(Q, z)$, 
TABLE II. Model parameters for $\frac{1}{2}^{+} \rightarrow \frac{1}{2}^{ \pm}$transitions.

\begin{tabular}{ll}
\hline \hline Transition & \multicolumn{1}{c}{ Choice of parameters } \\
\hline$N \rightarrow N(1650)$ & $c_{34}=-0.56, c_{45}=0.66, c_{56}=0.50$, \\
& $c_{44}=1.03, c_{55}=0.94$, \\
& $\eta=1.31, \zeta=-0.27, \xi=-0.03$ \\
$N \rightarrow N(1710)$ & $c_{33}=0.09, c_{44}=0.12, c_{55}=-0.05$, \\
& $g=1, \eta=-1.70, \lambda=0.95, \zeta=5.24, \xi=-8.22$ \\
$N \rightarrow \Delta(1620)$ & $c_{34}=-1.84, c_{45}=2.92, c_{56}=-0.73$, \\
& $c_{44}=-1.06, c_{55}=0.01$, \\
& $\eta=3.00, \zeta=-0.52, \xi=-0.10$ \\
\hline \hline
\end{tabular}

$V(Q, z)=\kappa^{2} z^{2} \int_{0}^{1} \frac{d x}{(1-x)^{2}} x^{a} e^{-\kappa^{2} z^{2} \frac{x}{1-x}}, \quad a=Q^{2} /\left(4 \kappa^{2}\right)$.

The sets of parameters $c_{\tau}, c_{\tau^{*}}$, and $g_{\tau \tau^{*}}^{(i M)}$ induce mixing of the contributions of AdS fields with different twist dimensions. The parameters $c_{\tau}$ and $c_{\tau^{*}}$ are constrained by the conditions $\sum_{\tau} c_{\tau}=1$ and $\sum_{\tau} c_{\tau^{*}}=1$, to guarantee the correct normalization of the kinetic terms $\bar{\psi}(x) i \not \partial \psi(x)$ of the four-dimensional spinor fields. This condition is also consistent with electromagnetic gauge invariance (see details in Refs. [48,58]). Therefore, the masses of the nucleon and nucleon resonance are identified by the expressions $[48,58]$

$M_{N}=2 \kappa \sum_{\tau} c_{\tau} \sqrt{\tau-1}, \quad M_{N^{*}}=2 \kappa \sum_{\tau} c_{\tau}^{*} \sqrt{\tau-1}$,

where the leading twist from which the sums start in Eq. (20) is defined as $\tau=3+L$, where $L$ is the angular orbital moment specified for baryons in Table I.

TABLE III. Model parameters for $\frac{1}{2}^{+} \rightarrow \frac{3}{2}^{ \pm}, \frac{5}{2}+$ transitions.

\begin{tabular}{|c|c|}
\hline Transition & Choice of parameters \\
\hline$N \rightarrow N(1520)$ & $\begin{array}{l}g_{34}^{1 A}=-13.79, g_{45}^{1 A}=-20.87, g_{56}^{1 A}=-8.70, g_{34}^{2 B}=1.01, g_{45}^{2 B}=-5.71, g_{56}^{2 B}=1.32 \\
g_{34}^{2 C}=-6.49, g_{45}^{2 C}=11.76, g_{56}^{2 C}=-5.04, g_{34}^{D D}=-17.46, g_{45}^{2 D}=26.44, g_{56}^{2 D}=-11.01 \\
g_{34}^{1 E}=-15.97, g_{45}^{1 E}=-90.70, g_{56}^{1 E}=20.76, g_{34}^{1 F}=-22.27, g_{45}^{1 F}=40.38, g_{56}^{1 F}=-17.29\end{array}$ \\
\hline$N \rightarrow N(1675)$ & $\begin{array}{l}g_{34}^{2 A}=-0.46, g_{45}^{2 A}=1.34, g_{56}^{2 A}=-0.77, g_{34}^{1 B}=-1.39, g_{45}^{1 B}=2.82, g_{56}^{1 B}=-6.60 \\
g_{34}^{1 C}=12.54, g_{45}^{1 C}=-51.12, g_{56}^{1 C}=42.51, g_{34}^{1 D}=-0.92, g_{45}^{1 D}=2.67, g_{56}^{1 D}=-1.53 \\
g_{34}^{2 E}=-0.70, g_{45}^{2 E}=1.42, g_{56}^{2 E}=-3.33, g_{34}^{2 E}=-0.55, g_{45}^{2 F}=2.23, g_{56}^{2 F}=-1.86\end{array}$ \\
\hline$N \rightarrow N(1680)$ & $\begin{array}{l}g_{35}^{1 A}=-2.64, g_{46}^{1 A}=2.98, g_{57}^{1 A}=-2.13, g_{35}^{2 B}=11.65, g_{46}^{2 B}=-13.13, g_{57}^{2 B}=9.39 \\
g_{35}^{2 C}=34.34, g_{46}^{2 C}=16.31, g_{57}^{2 C}=-21.05, g_{35}^{2 D}=11.65, g_{46}^{2 D}=-13.13, g_{57}^{2 D}=9.39 \\
g_{35}^{1 E}=-17.81, g_{46}^{1 E}=29.05, g_{57}^{1 E}=11.36, g_{35}^{1 F}=211.90, g_{46}^{1 F}=100.61, g_{57}^{1 F}=129.91\end{array}$ \\
\hline$N \rightarrow N(1700)$ & $\begin{array}{l}g_{34}^{1 A}=0.18, g_{45}^{1 A}=-0.28, g_{56}^{1 A}=0.16, g_{34}^{2 B}=-0.09, g_{45}^{2 B}=0.17, g_{56}^{2 B}=0.12 \\
g_{34}^{2 C}=0.02, g_{45}^{2 C}=0.06, g_{56}^{2 C}=-0.08, g_{34}^{2 D}=-0.20, g_{45}^{2 D}=0.31, g_{56}^{2 D}=-0.17 \\
g_{34}^{1 E}=1.48, g_{45}^{1 E}=-2.95, g_{56}^{1 E}=-2.03, g_{34}^{1 F}=-0.30, g_{45}^{1 F}=-1.06, g_{56}^{1 F}=1.46\end{array}$ \\
\hline$N \rightarrow N(1720)$ & $\begin{array}{l}g_{35}^{2 A}=-11.58, g_{46}^{2 A}=34.50, g_{57}^{2 A}=-22.60, g_{35}^{1 B}=79.15, g_{46}^{1 B}=67.11, g_{57}^{1 B}=29.95 \\
g_{35}^{1 C}=-36.53, g_{46}^{1 C}=105.65, g_{57}^{1 C}=-58.59, g_{35}^{1 D}=0.16, g_{46}^{1 D}=-0.47, g_{57}^{1 D}=0.31 \\
g_{35}^{2 E}=12.14, g_{46}^{2 E}=10.29, g_{57}^{2 E}=4.59, g_{35}^{2 F}=5.56, g_{46}^{2 F}=-16.07, g_{57}^{2 F}=8.91\end{array}$ \\
\hline$N \rightarrow N^{\prime}(1720)$ & $\begin{array}{l}g_{35}^{2 A}=30.18, g_{46}^{2 A}=-54.02, g_{57}^{2 A}=24.82, g_{35}^{1 B}=-1.15, g_{46}^{1 B}=6.41, g_{57}^{1 B}=-2.87 \\
g_{35}^{1 C}=-17.77, g_{46}^{1 C}=107.19, g_{57}^{1 C}=-62.71, g_{35}^{1 D}=8.48, g_{46}^{1 D}=-15.18, g_{57}^{1 D}=6.97 \\
g_{35}^{2 E}=-0.60, g_{46}^{2 E}=3.37, g_{57}^{2 E}=-1.51, g_{35}^{2 F}=-3.57, g_{46}^{2 F}=21.56, g_{57}^{2 F}=-12.61\end{array}$ \\
\hline$N \rightarrow \Delta(1232)$ & $\begin{array}{l}g_{33}^{2 A}=-14.00, g_{44}^{2 A}=22.38, g_{55}^{2 A}=-10.13, g_{33}^{1 B}=-0.26, g_{44}^{1 B}=0.18, g_{55}^{1 B}=2.05 \\
g_{33}^{1 C}=0.97, g_{44}^{1 C}=-3.01, g_{55}^{1 C}=2.05, g_{33}^{1 D}=-6.49, g_{44}^{1 D}=10.39, g_{55}^{1 D}=-4.70 \\
g_{33}^{2 E}=-3.82, g_{44}^{2 E}=0.26, g_{55}^{2 E}=2.97, g_{33}^{2 F}=0.57, g_{44}^{2 F}=-1.77, g_{55}^{2 F}=1.20\end{array}$ \\
\hline$N \rightarrow \Delta(1700)$ & $\begin{array}{l}g_{34}^{1 A}=-0.01, g_{45}^{1 A}=-0.59, g_{56}^{1 A}=0.40, g_{34}^{2 B}=-0.53, g_{45}^{2 B}=0.70, g_{56}^{2 B}=-0.27 \\
g_{34}^{2 C}=1.02, g_{45}^{2 C}=2.70, g_{56}^{2 C}=-0.97, g_{34}^{2 D}=-0.05, g_{45}^{2 D}=3.57, g_{56}^{2 D}=-2.44 \\
g_{34}^{1 E}=-0.93, g_{45}^{1 E}=1.23, g_{56}^{1 E}=-0.47, g_{34}^{1 F}=12.09, g_{45}^{1 F}=32.11, g_{56}^{1 F}=-11.49\end{array}$ \\
\hline
\end{tabular}



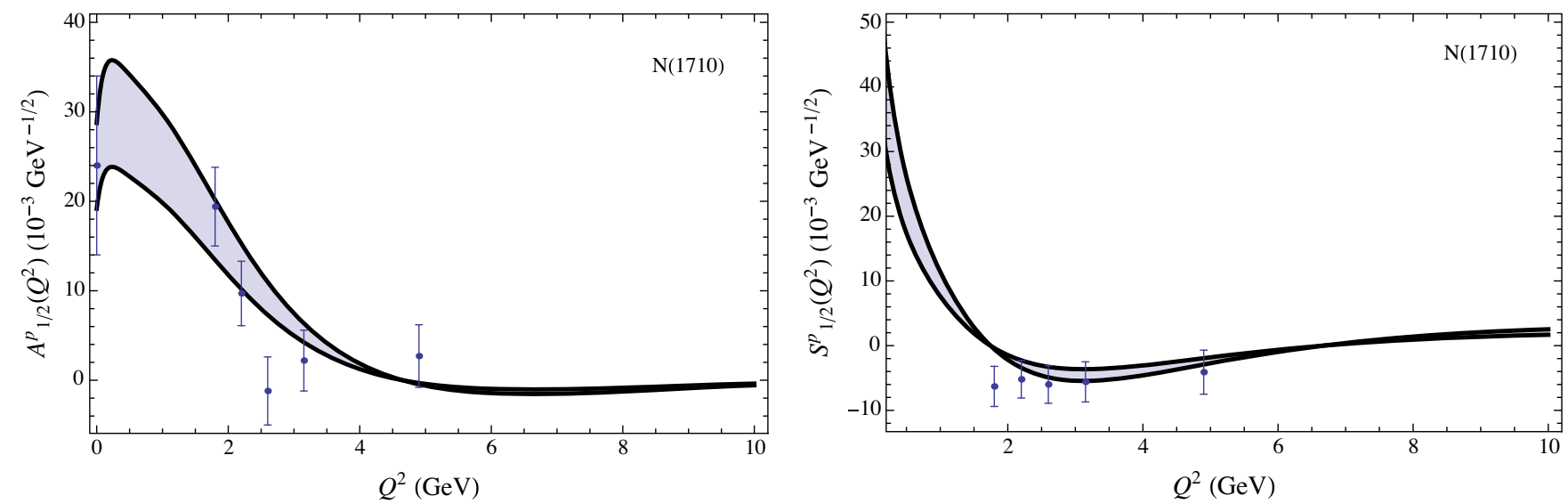

FIG. 1. Helicity amplitudes $A_{1 / 2}^{p}\left(Q^{2}\right)$ (left panel) and $S_{1 / 2}^{p}\left(Q^{2}\right)$ (right panel) for $N \gamma^{*} \rightarrow N(1710)$ transition up to $Q^{2}=10 \mathrm{GeV}$. Our results are shown with a variation of the parameters of our approach (shaded band), and comparing with data taken from the CLAS Collaboration [68]. Here and in the following superscript $(p)$ in the notation of the helicity amplitudes means the proton channel.
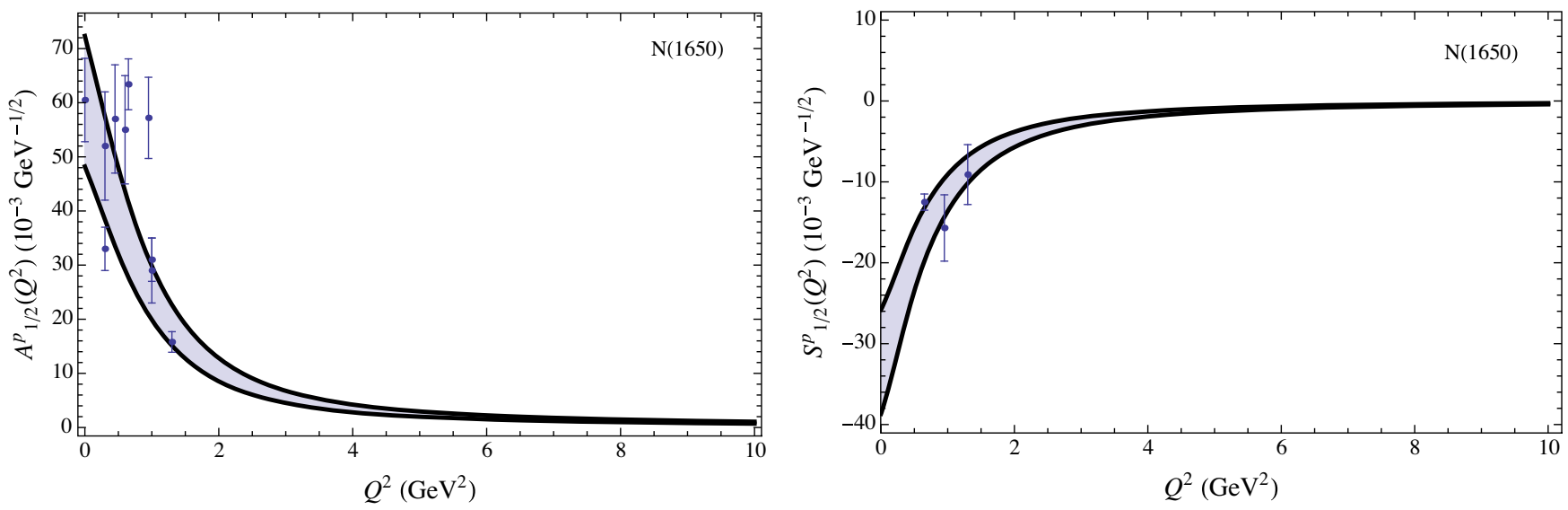

FIG. 2. Helicity amplitudes $A_{1 / 2}^{p}\left(Q^{2}\right)$ (left panel) and $S_{1 / 2}^{p}\left(Q^{2}\right)$ (right panel) for $N \gamma^{*} \rightarrow N(1650)$ transition up to $Q^{2}=10$ GeV ${ }^{2}$ Our results are shown with a variation of the parameters of our approach (shaded band) comparing with data taken from the CLAS Collaboration [69,70] and compilation of the world analyses of the $N \pi$ electroproduction data [81].
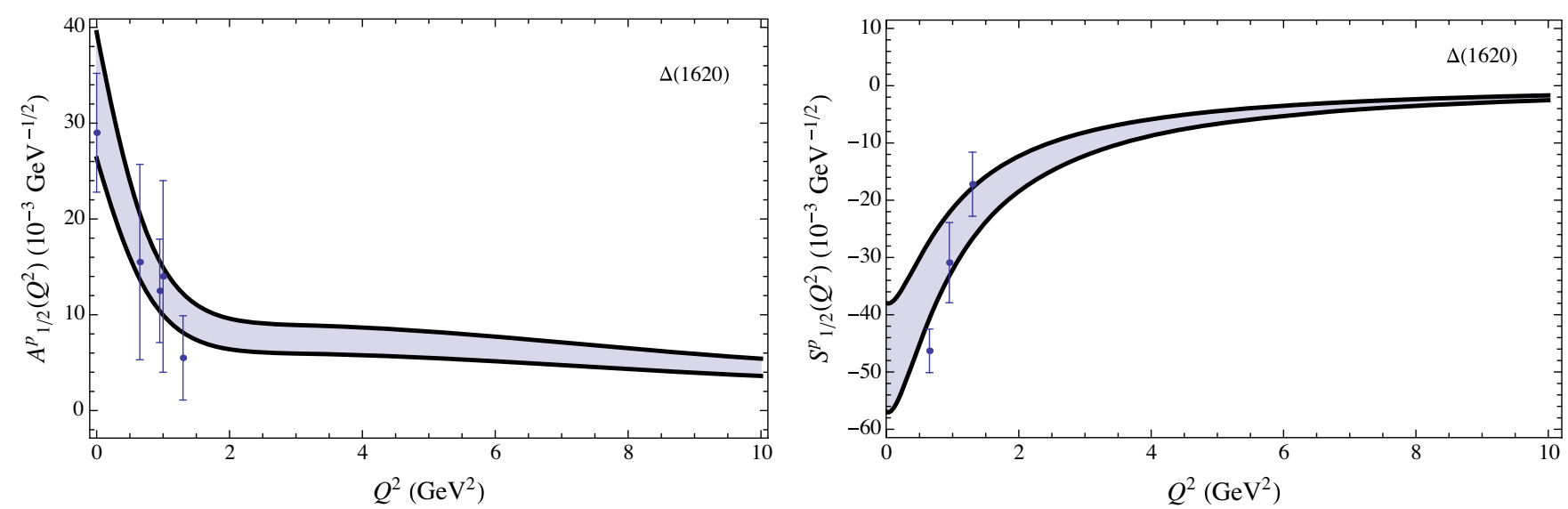

FIG. 3. Helicity amplitudes $A_{1 / 2}^{p}\left(Q^{2}\right)$ (left panel) and $S_{1 / 2}^{p}\left(Q^{2}\right)$ (right panel) for $N \gamma^{*} \rightarrow \Delta(1620)$ transition up to $Q^{2}=10 \mathrm{GeV}$. Our results (shaded band) are compared with data taken from the CLAS Collaboration $[6,69]$, a compilation of the world analyses of $N \pi$ electroproduction data [81] and Particle Data Group (PDG) [63]. 

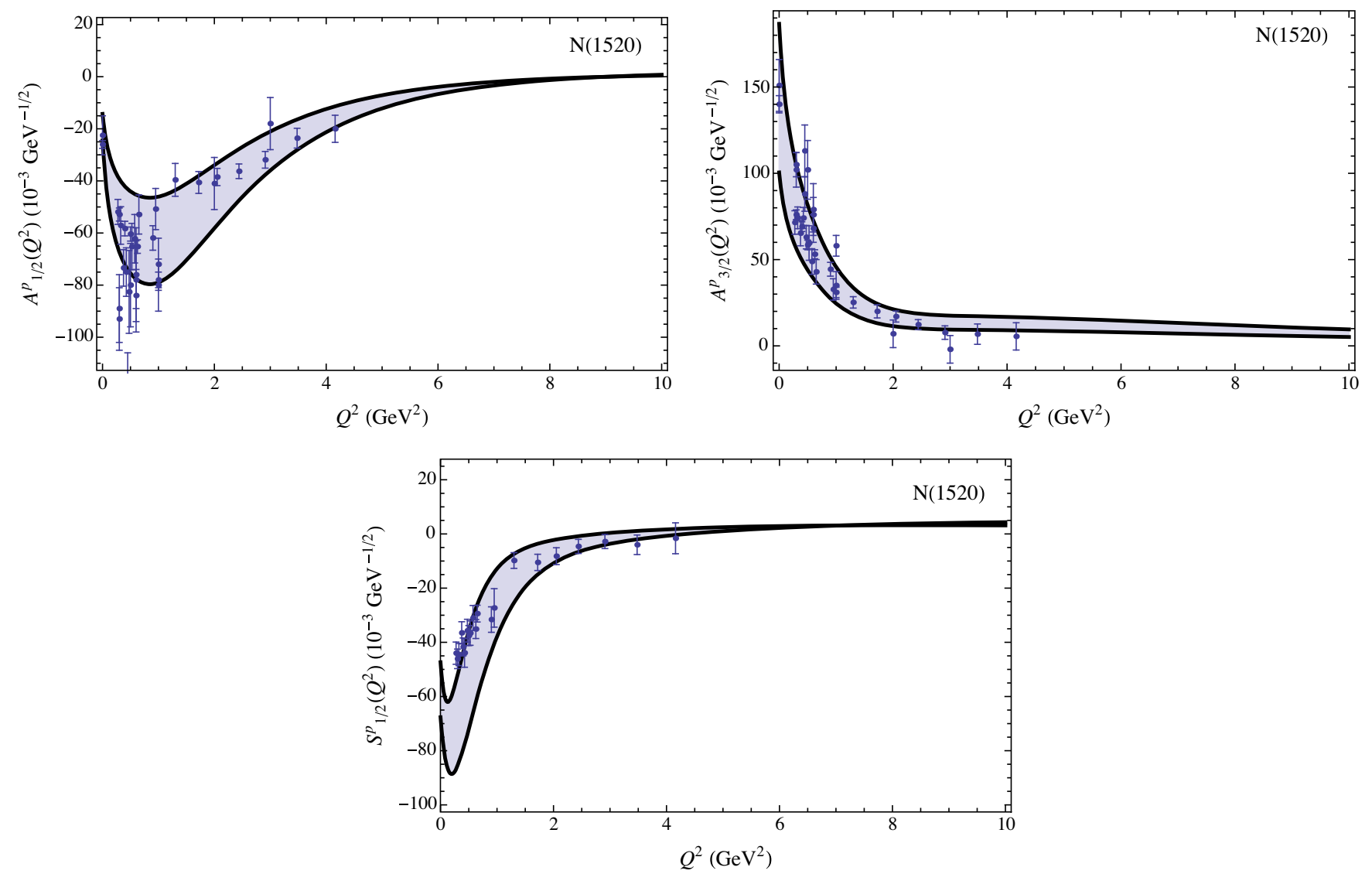

FIG. 4. Helicity amplitudes $A_{1 / 2}^{p}\left(Q^{2}\right)$ (left upper panel), $A_{3 / 2}^{p}\left(Q^{2}\right)$ (right upper panel), and $S_{1 / 2}^{p}\left(Q^{2}\right)$ (centered lower panel), for $N \gamma^{*} \rightarrow N(1520)$ transition up to $Q^{2}=10 \mathrm{GeV}^{2}$. Our results (shaded band) are compared with data taken from the CLAS Collaboration $[4,6,71,72]$, a compilation of data [81], and PDG [63].

The baryon form factors are determined analytically using the bulk profiles of fermion fields and the bulk-toboundary propagator $V(Q, z)$ of the vector field (for exact expressions see the next section). The calculational technique was already described in detail in Refs. $[48,51,58]$. The parameter $\kappa=383 \mathrm{MeV}$ is universal and was fixed in previous studies (see, e.g., Refs. [48,58]), while the other parameters are fixed from a fit to the helicity amplitudes of the $\gamma^{*} N \rightarrow N^{*}$ transitions.

\section{ELECTROMAGNETIC FORM FACTORS AND HELICITY AMPLITUDES OF THE $\gamma^{*} N \rightarrow N^{*}$ TRANSITIONS}

Due to Lorenz covariance and gauge invariance, the matrix elements of the electromagnetic $\gamma^{*} N \rightarrow N^{*}$ transitions can be expressed in terms of their general Lorenz structures as

$$
H_{\mu \nu}^{(1)}=\not g g_{\mu \nu}-\gamma_{\mu} q_{\nu}, \quad H_{\mu \nu}^{(2)}=p_{1 \mu} q_{\nu}-g_{\mu \nu} p_{1} q, \quad H_{\mu \nu}^{(3)}=q_{\mu} q_{\nu}-g_{\mu \nu} q^{2}
$$

and the relativistic form factors $G_{i}\left(Q^{2}\right), i=1,2,3$ as $[1-3,66,67]$

$$
\begin{aligned}
\left\langle N^{*}\left|J_{\mu}^{\mathrm{em}}\right| N\right\rangle & =\bar{u}_{N^{*}}^{\nu \nu_{1} \ldots \nu_{l-1}}\left(p_{1} \lambda_{1}\right) q_{\nu_{1}} \ldots q_{\nu_{l-1}}\left(\begin{array}{c}
-\gamma_{5} \\
I
\end{array}\right) \Gamma_{\mu \nu}\left(Q^{2}\right) u_{N}\left(p_{2} \lambda_{2}\right), \\
\Gamma_{\mu \nu}\left(Q^{2}\right) & =G_{1}\left(Q^{2}\right) H_{\mu \nu}^{(1)}+G_{2}\left(Q^{2}\right) H_{\mu \nu}^{(2)}+G_{3}\left(Q^{2}\right) H_{\mu \nu}^{(3)} .
\end{aligned}
$$

Here $u_{N}\left(p_{2} \lambda_{2}\right)$ and $u_{N^{*}}^{\nu \nu_{1} \ldots \nu_{l-1}}\left(p_{1} \lambda_{1}\right)$ are spin- $\frac{1}{2}$ and higher spin (Rarita-Schwinger) spinors, respectively. The RaritaSchwinger spinor satisfies the conditions

$$
\bar{u}_{N^{*}}^{\nu \nu_{1} \ldots \nu_{l-1}} \gamma_{\alpha}=\bar{u}_{N^{*}}^{\nu \nu_{1} \ldots \nu_{l-1}} q_{\alpha}=0 \quad \text { for } \alpha \in\left\{\nu, \nu_{1}, \ldots, \nu_{l-1}\right\}
$$



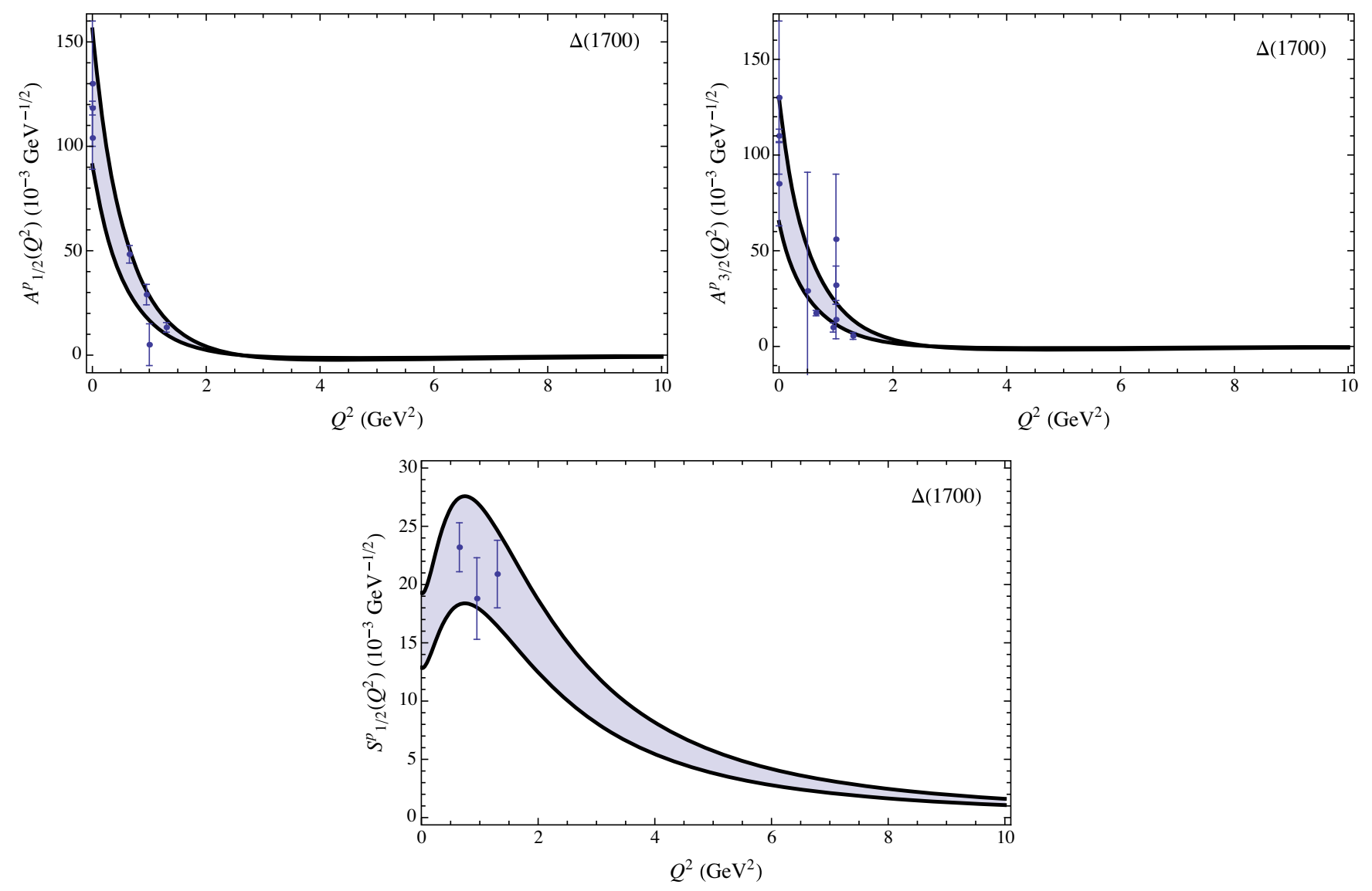

FIG. 5. Helicity amplitudes $A_{1 / 2}^{p}\left(Q^{2}\right)$ (left upper panel), $A_{3 / 2}^{p}\left(Q^{2}\right)$ (right upper panel), and $S_{1 / 2}^{p}\left(Q^{2}\right)$ (centered lower panel), for $N \gamma^{*} \rightarrow \Delta(1700)$ transition up to $Q^{2}=10 \mathrm{GeV}^{2}$. Our results (shaded band) are compared with data taken from the CLAS Collaboration [70,71], a compilation of data [81], and PDG [63].

$q=p_{1}-p_{2}$, and $\lambda_{1}, \lambda_{2}$, and $\lambda$ are the helicities of the final, initial baryon, and photon, respectively, with the relation $\lambda_{2}=\lambda_{1}-\lambda$. In the rest frame of the $N^{*}$ the four momenta of $N^{*}, N$, photon and the polarization vector of photon are specified as

$$
\begin{aligned}
p_{1} & =\left(M_{1}, \overrightarrow{0}\right), \quad p_{2}=(E, 0,0,-|\mathbf{p}|), \quad q=\left(q^{0}, 0,0,|\mathbf{p}|\right), \\
\epsilon^{\mu}( \pm) & =\left(0,-\vec{\epsilon}^{ \pm}\right), \quad \vec{\epsilon}( \pm)=\frac{1}{\sqrt{2}}( \pm 1, i, 0), \quad \epsilon^{\mu}(0)=\frac{1}{\sqrt{Q^{2}}}\left(|\mathbf{p}|, 0,0, q^{0}\right),
\end{aligned}
$$

where $|\mathbf{p}|=\frac{\sqrt{Q_{+} Q_{-}}}{2 M_{1}}$ is the absolute value of the three-momentum of the nucleon or the photon, $Q_{ \pm}=M_{ \pm}^{2}+Q^{2}$, and $M_{ \pm}=M_{1} \pm M_{2}$.

It is convenient to introduce the helicity amplitudes $A_{1 / 2}, A_{3 / 2}$, and $S_{1 / 2}$ responsible for the helicity transitions $\lambda_{2}= \pm \frac{1}{2} \rightarrow \lambda_{1}=\mp \frac{1}{2}, \lambda_{2}= \pm \frac{1}{2} \rightarrow \lambda_{1}= \pm \frac{3}{2}$, and $\lambda_{2}= \pm \frac{1}{2} \rightarrow \lambda_{1}= \pm \frac{1}{2}$, respectively, which are related to the invariant form factors $G_{i}\left(Q^{2}\right)$ as $[1-3]$

$$
A_{1 / 2}\left(Q^{2}\right)=b h_{3}\left(Q^{2}\right), \quad A_{3 / 2}\left(Q^{2}\right)= \pm \frac{b \sqrt{3}}{l} h_{2}\left(Q^{2}\right), \quad S_{1 / 2}\left(Q^{2}\right)=\frac{b|\mathbf{p}|}{M_{1} \sqrt{2}} h_{1}\left(Q^{2}\right),
$$

where

$$
\begin{aligned}
& h_{1}\left(Q^{2}\right)= \pm 4 M_{1} G_{1}\left(Q^{2}\right)+4 M_{1}^{2} G_{2}\left(Q^{2}\right)+2\left(M_{+} M_{-}-Q^{2}\right) G_{3}\left(Q^{2}\right), \\
& h_{2}\left(Q^{2}\right)=\mp 2 M_{ \pm} G_{1}\left(Q^{2}\right)-\left(M_{+} M_{-}-Q^{2}\right) G_{2}\left(Q^{2}\right)+2 Q^{2} G_{3}\left(Q^{2}\right), \\
& h_{3}\left(Q^{2}\right)=-\frac{2}{M_{1}}\left(M_{ \pm} M_{2} \pm Q^{2}\right) G_{1}\left(Q^{2}\right)+\left(M_{+} M_{-}-Q^{2}\right) G_{2}\left(Q^{2}\right)-2 Q^{2} G_{3}\left(Q^{2}\right),
\end{aligned}
$$



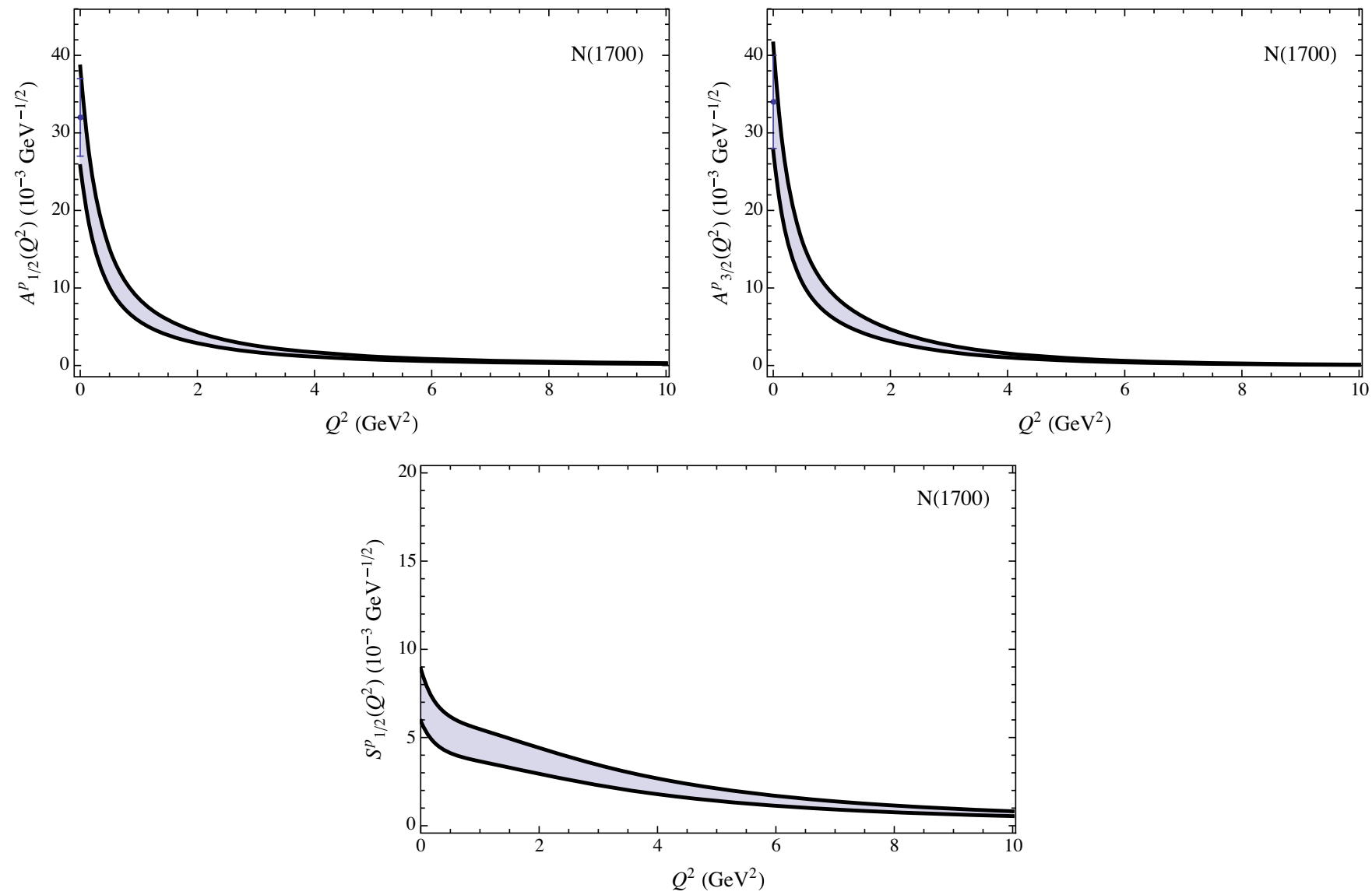

FIG. 6. Helicity amplitudes $A_{1 / 2}^{p}\left(Q^{2}\right)$ (left upper panel), $A_{3 / 2}^{p}\left(Q^{2}\right)$ (right upper panel), and $S_{1 / 2}^{p}\left(Q^{2}\right)$ (centered lower panel), for $N \gamma^{*} \rightarrow N(1700)$ transition up to $Q^{2}=10 \mathrm{GeV}^{2}$. Our results (shaded band) are compared with PDG [63].

$$
b=|\mathbf{p}|^{l-1} \sqrt{\frac{\pi \alpha Q_{\mp}}{8 M_{+} M_{-} M_{2} c_{l+1}}}, \quad c_{l}=\frac{(2 l) !}{2^{l}(l !)^{2}}, \quad l=J-\frac{1}{2},
$$

where $\alpha=1 / 137.036$ is the fine-structure constant.

The relations expressing the relativistic form factors $G_{i}$ through the set of form factors $h_{i}$ and helicity amplitudes read

$$
\begin{aligned}
G_{1}\left(Q^{2}\right) & =\mp \frac{h_{2}\left(Q^{2}\right)+h_{3}\left(Q^{2}\right)}{2 Q_{+}} M_{1}=\mp \frac{M_{1}}{2 Q_{ \pm} b}\left[A_{1 / 2}\left(Q^{2}\right) \pm \frac{l}{\sqrt{3}} A_{3 / 2}\left(Q^{2}\right)\right], \\
G_{2}\left(Q^{2}\right) & =\frac{1}{Q_{+} Q_{-}}\left[h_{1}\left(Q^{2}\right) Q^{2}+h_{2}\left(Q^{2}\right)\left(Q^{2} \mp M_{\mp} M_{2}\right)+h_{3}\left(Q^{2}\right) M_{\mp} M_{1}\right] \\
& =\frac{1}{Q_{+} Q_{-} b}\left[S_{1 / 2}\left(Q^{2}\right) \frac{Q^{2} M_{1} \sqrt{2}}{|\mathbf{p}|} \pm \frac{l}{\sqrt{3}} A_{3 / 2}\left(Q^{2}\right)\left(Q^{2} \mp M_{\mp} M_{2}\right)+A_{1 / 2}\left(Q^{2}\right) M_{\mp} M_{1}\right], \\
G_{3}\left(Q^{2}\right) & =\frac{1}{Q_{+} Q_{-}}\left[h_{1}\left(Q^{2}\right)\left(M_{+} M_{-}-Q^{2}\right)+\left(h_{2}\left(Q^{2}\right)-h_{3}\left(Q^{2}\right)\right) M_{1}^{2}\right] \\
& =\frac{1}{Q_{+} Q_{-} b}\left[S_{1 / 2}\left(Q^{2}\right) \frac{M_{1}}{|\mathbf{p}| \sqrt{2}}\left(M_{+} M_{-}-Q^{2}\right)+\left( \pm \frac{l}{\sqrt{3}} A_{3 / 2}\left(Q^{2}\right)-A_{1 / 2}\left(Q^{2}\right)\right) M_{1}^{2}\right] .
\end{aligned}
$$

The structure of the $\frac{1}{2}^{+} \gamma^{*} \rightarrow \frac{1}{2}^{ \pm}$is simpler and is given by the form

$$
M^{\mu}\left(p_{1} \lambda_{1}, p_{2} \lambda_{2}\right)=\bar{u}_{N^{*}}\left(p_{1} \lambda_{1}\right)\left[\gamma_{\perp}^{\mu} F_{1}^{N^{*} N}\left(-q^{2}\right)+i \sigma^{\mu \nu} \frac{q_{\nu}}{M_{+}} F_{2}^{N^{*} N}\left(-q^{2}\right)\right]\left(\begin{array}{c}
I \\
\gamma_{5}
\end{array}\right) u_{N}\left(p_{2} \lambda_{2}\right)
$$

where $\gamma_{\perp}^{\mu}=\gamma^{\mu}-q^{\mu} \not / / q^{2}$. 

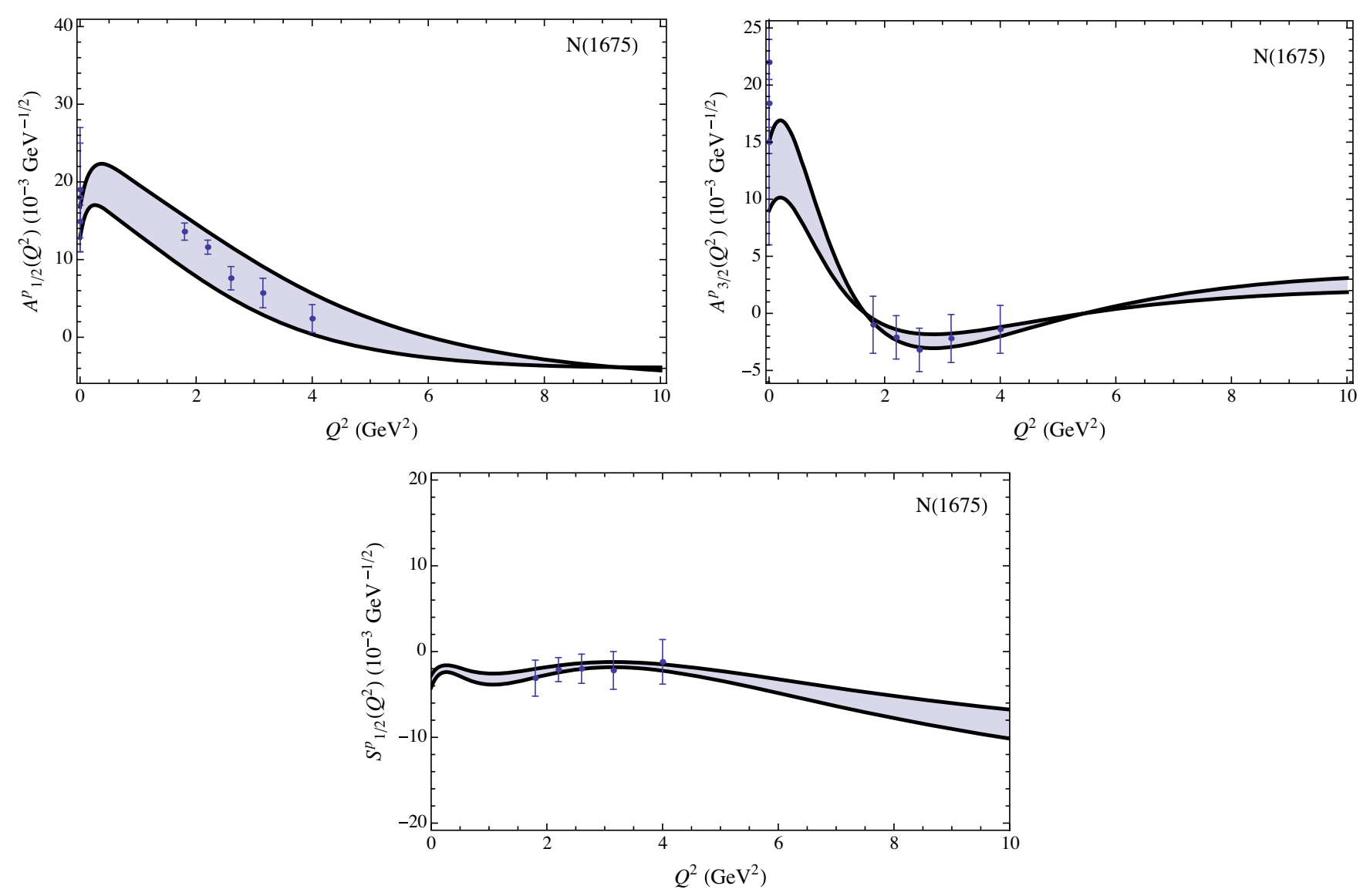

FIG. 7. Helicity amplitudes $A_{1 / 2}^{p}\left(Q^{2}\right)$ (left upper panel), $A_{3 / 2}^{p}\left(Q^{2}\right)$ (right upper panel), and $S_{1 / 2}^{p}\left(Q^{2}\right)$ (centered lower panel), for $N \gamma^{*} \rightarrow N(1675)$ transition up to $Q^{2}=10 \mathrm{GeV}^{2}$. Our results (shaded band) are compared with data taken from the CLAS Collaboration $[68,71]$ and PDG [63].

The helicity amplitudes defining the $\frac{1}{2}^{+} \rightarrow \frac{1}{2}^{+}$and $\frac{1}{2}^{+} \rightarrow \frac{1}{2}^{-}$ transitions in terms of form factors are defined, respectively, as

$$
\begin{aligned}
A_{1 / 2}^{ \pm}\left(Q^{2}\right)= & \sqrt{\frac{2 \pi \alpha Q_{\mp}}{M_{1} M_{2} E}}\left[F_{1}^{N^{*} N}\left(Q^{2}\right)+F_{2}^{N^{*} N}\left(Q^{2}\right) \frac{M_{ \pm}}{M_{+}}\right], \\
S_{1 / 2}^{ \pm}\left(Q^{2}\right)= & \pm \frac{|\mathbf{p}|}{M_{+}} \sqrt{\frac{\pi \alpha Q_{\mp}}{M_{1} M_{2} E}} \\
& \times\left[F_{1}^{N^{*} N}\left(Q^{2}\right) \frac{M_{ \pm} M_{+}}{Q^{2}}-F_{2}^{N^{*} N}\left(Q^{2}\right)\right],
\end{aligned}
$$

where $E=M_{+} M_{-} /\left(2 M_{1}\right)$.

In the case of the high-spin resonances, the set of helicity amplitudes $\left(A_{1 / 2}, A_{3 / 2}, S_{1 / 2}\right)$ is related to the set of the charge $\left(G_{E}\right)$, magnetic $\left(G_{M}\right)$, and Coulomb $\left(G_{Q}\right)$ form factors [1-3]:

$$
\begin{aligned}
G_{E} & =-F_{l}^{+} \frac{2}{l+1}\left(\frac{l}{\sqrt{3}} A_{3 / 2}-A_{1 / 2}\right), \\
G_{M} & =-F_{l}^{+} \frac{2 l}{l+1}\left(\frac{l+2}{\sqrt{3}} A_{3 / 2}+A_{1 / 2}\right), \\
G_{C} & =2 \sqrt{2} F_{l}^{+} \frac{M_{1}}{|\mathbf{p}|} S_{1 / 2}
\end{aligned}
$$

for abnormal parity transitions $\frac{1}{2}^{+} \rightarrow \frac{3}{2}^{+}, \frac{5}{2}^{-}, \ldots$

$$
\begin{aligned}
G_{E} & =-F_{l}^{-} \frac{2 l}{l+1}\left(\frac{l+2}{\sqrt{3}} A_{3 / 2}+A_{1 / 2}\right), \\
G_{M} & =-F_{l}^{-} \frac{2}{l+1}\left(\frac{l}{\sqrt{3}} A_{3 / 2}-A_{1 / 2}\right), \\
G_{C} & =2 \sqrt{2} F_{l}^{-} \frac{M_{1}}{|\mathbf{p}|} S_{1 / 2}
\end{aligned}
$$

for normal parity transitions $\frac{1}{2}^{+} \rightarrow \frac{3}{2}^{-}, \frac{5}{2}^{+}, \ldots$

In terms of the relativistic form factor $h_{i}$, the charge, magnetic, and Coulomb form factors are expressed as

$$
\begin{aligned}
G_{E} & =-\frac{2 b F_{l}^{+}}{l+1}\left(h_{2}-h_{3}\right), \\
G_{M} & =-\frac{2 b F_{l}^{+}}{l+1}\left[l\left(h_{2}+h_{3}\right)+2 h_{2}\right], \\
G_{E} & =2 b F_{l}^{+} h_{1}
\end{aligned}
$$

for abnormal parity transitions and 

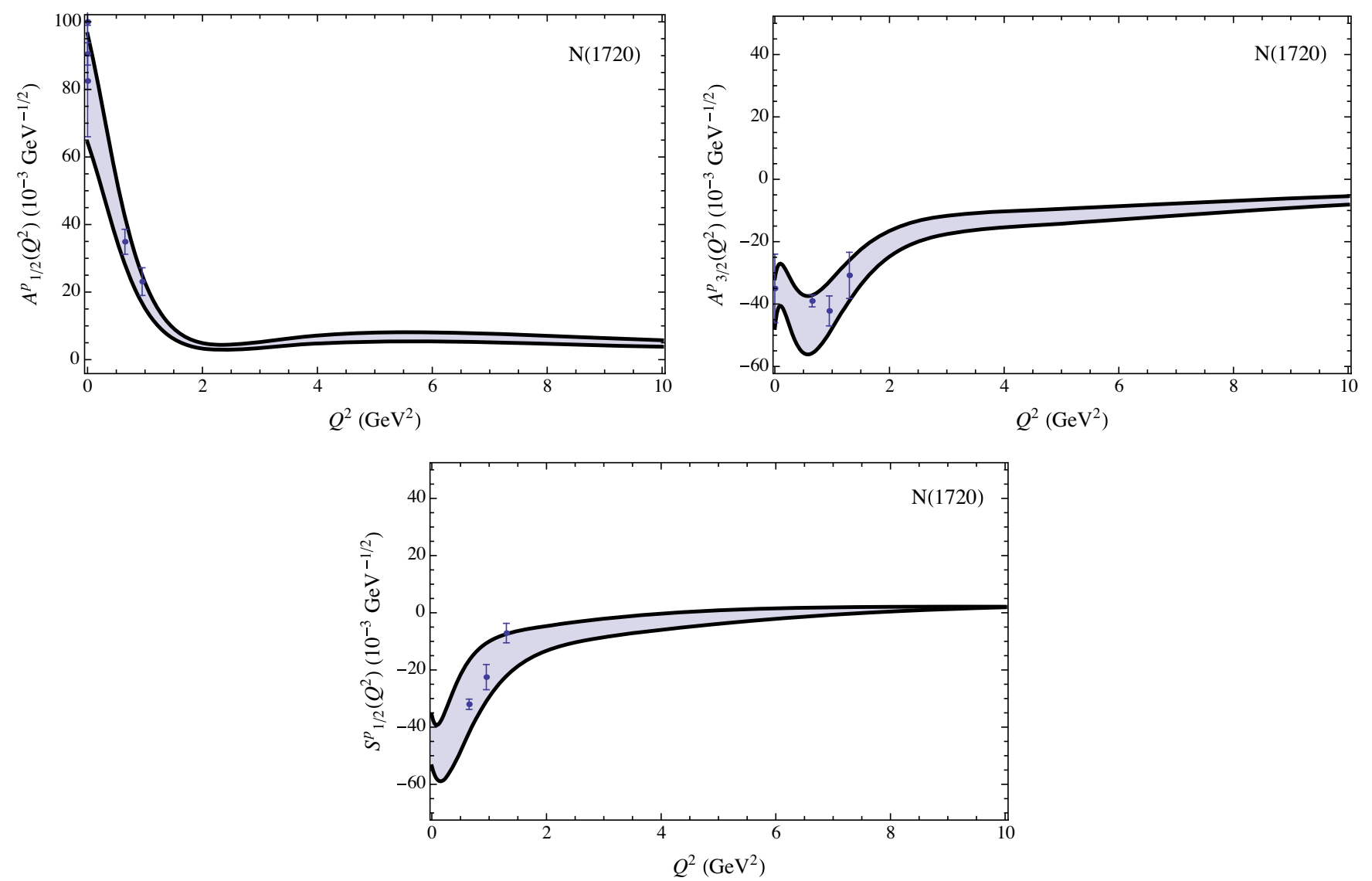

FIG. 8. Helicity amplitudes $A_{1 / 2}^{p}\left(Q^{2}\right)$ (left upper panel), $A_{3 / 2}^{p}\left(Q^{2}\right)$ (right upper panel), and $S_{1 / 2}^{p}\left(Q^{2}\right)$ (centered lower panel), for $N \gamma^{*} \rightarrow N(1720)$ transition up to $Q^{2}=10 \mathrm{GeV}^{2}$. Our results (shaded band) are compared with data taken from the CLAS Collaboration [64] and PDG [63].

$$
\begin{aligned}
G_{E} & =\frac{2 b F_{l}^{-}}{l+1}\left[l\left(h_{2}-h_{3}\right)+2 h_{2}\right], \\
G_{M} & =-\frac{2 b F_{l}^{-}}{l+1}\left(h_{2}-h_{3}\right), \\
G_{E} & =2 b F_{l}^{-} h_{1}
\end{aligned}
$$

for normal parity transitions. Here

$$
F_{l}^{ \pm}=\frac{M_{2}}{|\mathbf{p}|^{l}} \sqrt{c_{l+1} \frac{M_{2} E}{6 \pi \alpha M_{1}} \frac{Q_{ \pm}}{M_{ \pm}^{2}}} .
$$

The form factors $G_{i}^{(n)}\left(Q^{2}\right)$ (here $n$ is the radial quantum number) defining the abnormal parity transitions are given by

$$
\begin{aligned}
G_{1}^{(n)}\left(Q^{2}\right)= & \sum_{\tau \tau^{*}}\left[g_{\tau \tau^{*}}^{(2 A)} L_{1-\tau \tau^{*}}^{(n)}\left(Q^{2}\right)-g_{\tau \tau^{*}}^{(1 D)} L_{1+\tau \tau^{*}}^{(n)}\left(Q^{2}\right)+g_{\tau \tau^{*}}^{(3 A)} L_{2+\tau \tau^{*}}^{(n)}\left(Q^{2}\right)-g_{\tau \tau^{*}}^{(4 D)} L_{2-\tau \tau^{*}}^{(n)}\left(Q^{2}\right)\right], \\
G_{2}^{(n)}\left(Q^{2}\right)= & \frac{1}{\kappa} \sum_{\tau \tau^{*}}\left[-g_{\tau \tau^{*}}^{(1 B)} L_{2-\tau \tau^{*}}^{(n)}\left(Q^{2}\right)+g_{\tau \tau^{*}}^{(4 B)} L_{1+\tau \tau^{*}}^{(n)}\left(Q^{2}\right)+g_{\tau \tau^{*}}^{(2 E)} L_{2+\tau \tau^{*}}^{(n)}\left(Q^{2}\right)-g_{\tau \tau^{*}}^{(3 E)} L_{1-\tau \tau^{*}}^{(n)}\left(Q^{2}\right)\right], \\
G_{3}^{(n)}\left(Q^{2}\right)= & \frac{1}{\kappa} \sum_{\tau \tau^{*}}\left[g_{\tau \tau^{*}}^{(2 A)} M_{2+\tau \tau^{*}}^{(n)}\left(Q^{2}\right)-g_{\tau \tau^{*}}^{(1 D)} M_{2-\tau \tau^{*}}^{(n)}\left(Q^{2}\right)-g_{\tau \tau^{*}}^{(3 A)} M_{1-\tau \tau^{*}}^{(n)}\left(Q^{2}\right)+g_{\tau \tau^{*}}^{(4 D)} M_{1+\tau \tau^{*}}^{(n)}\left(Q^{2}\right)\right. \\
& +g_{\tau \tau^{*}}^{(1 B)} K_{2-\tau \tau^{*}}^{(n)}\left(Q^{2}\right)-g_{\tau \tau^{*}}^{(4 B)} K_{1+\tau \tau^{*}}^{(n)}\left(Q^{2}\right)-g_{\tau \tau^{*}}^{(2 E)} K_{2+\tau \tau^{*}}^{(n)}\left(Q^{2}\right)+g_{\tau^{*}}^{(3 E)} K_{1-\tau \tau^{*}}^{(n)}\left(Q^{2}\right) \\
& \left.+g_{\tau \tau^{*}}^{(1 C)} R_{2-\tau \tau^{*}}^{(n)}\left(Q^{2}\right)-g_{\tau \tau^{*}}^{(4 C)} R_{1+\tau \tau^{*}}^{(n)}\left(Q^{2}\right)-g_{\tau \tau^{*}}^{(2 F)} R_{2+\tau \tau^{*}}^{(n)}\left(Q^{2}\right)+g_{\tau \tau^{*}}^{(3 F)} R_{1-\tau \tau^{*}}^{(n)}\left(Q^{2}\right)\right],
\end{aligned}
$$



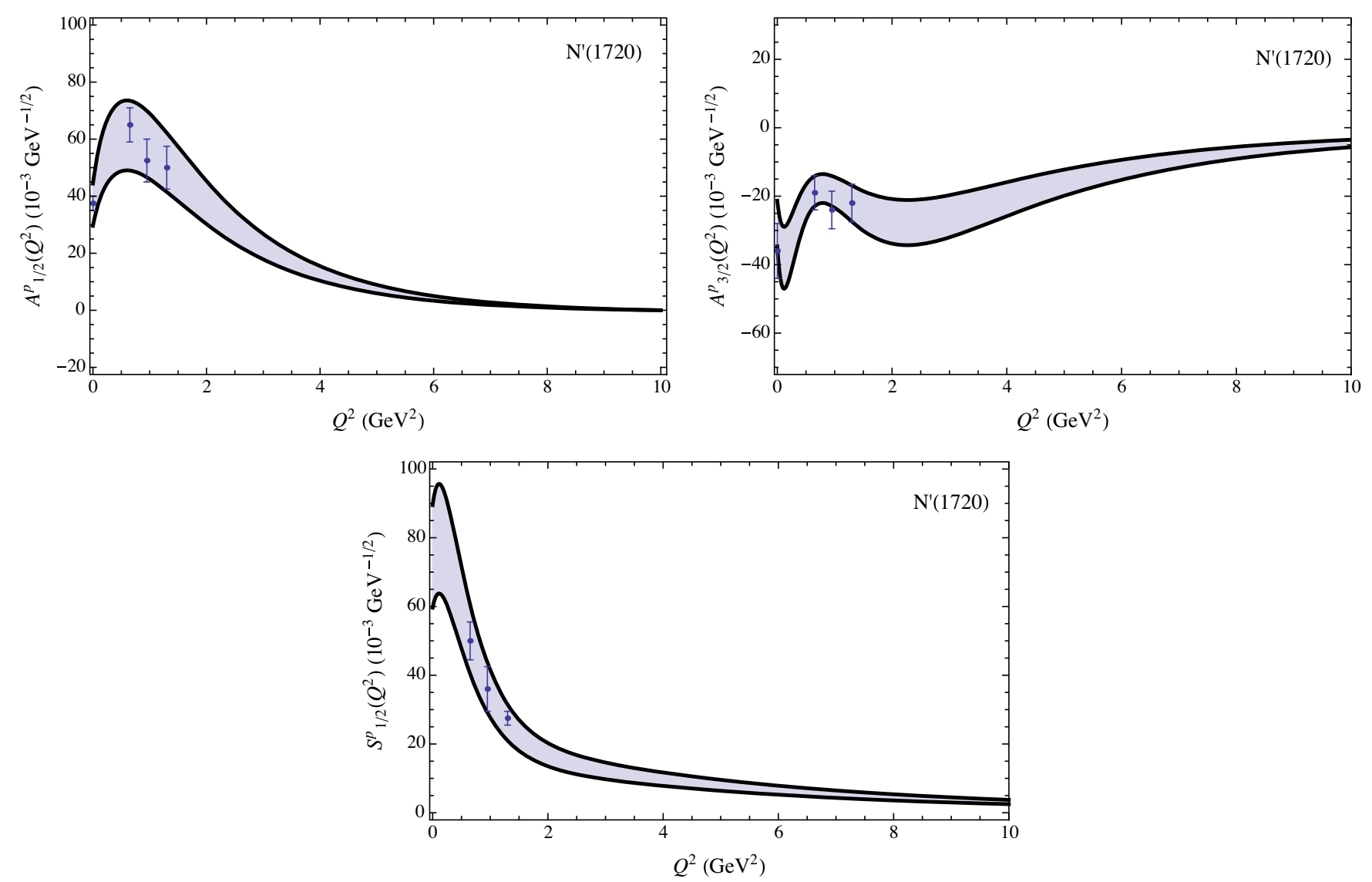

FIG. 9. Helicity amplitudes $A_{1 / 2}^{p}\left(Q^{2}\right)$ (left upper panel), $A_{3 / 2}^{p}\left(Q^{2}\right)$ (right upper panel), and $S_{1 / 2}^{p}\left(Q^{2}\right)$ (centered lower panel), for $N \gamma^{*} \rightarrow N^{\prime}(1720)$ transition up to $Q^{2}=10 \mathrm{GeV}^{2}$. Our results (shaded band) are compared with data taken from the CLAS Collaboration [64] and PDG [63].

$$
\begin{aligned}
G_{1}^{(n)}\left(Q^{2}\right)= & \sum_{\tau \tau^{*}}\left[g_{\tau \tau^{*}}^{(1 A)} L_{1+\tau \tau^{*}}^{(n)}\left(Q^{2}\right)-g_{\tau \tau^{*}}^{(2 D)} L_{1-\tau \tau^{*}}^{(n)}\left(Q^{2}\right)+g_{\tau \tau^{*}}^{(4 A)} L_{2-\tau \tau^{*}}^{(n)}\left(Q^{2}\right)-g_{\tau \tau^{*}}^{(3 D)} L_{2+\tau \tau^{*}}^{(n)}\left(Q^{2}\right)\right], \\
G_{2}^{(n)}\left(Q^{2}\right)= & \frac{1}{\kappa} \sum_{\tau \tau^{*}}\left[-g_{\tau \tau^{*}}^{(2 B)} L_{2+\tau \tau^{*}}^{(n)}\left(Q^{2}\right)+g_{\tau \tau^{*}}^{(3 B)} L_{1-\tau \tau^{*}}^{(n)}\left(Q^{2}\right)+g_{\tau \tau^{*}}^{(1 E)} L_{2-\tau \tau^{*}}^{(n)}\left(Q^{2}\right)-g_{\tau \tau^{*}}^{(4 E)} L_{1+\tau \tau^{*}}^{(n)}\left(Q^{2}\right)\right], \\
G_{3}^{(n)}\left(Q^{2}\right)= & \frac{1}{\kappa} \sum_{\tau \tau^{*}}\left[g_{\tau \tau^{*}}^{(1 A)} M_{2-\tau \tau^{*}}^{(n)}\left(Q^{2}\right)-g_{\tau \tau^{*}}^{(2 D)} M_{2+\tau \tau^{*}}^{(n)}\left(Q^{2}\right)-g_{\tau \tau^{*}}^{(4 A)} M_{1+\tau \tau^{*}}^{(n)}\left(Q^{2}\right)+g_{\tau \tau^{*}}^{(3 D)} M_{1-\tau \tau^{*}}^{(n)}\left(Q^{2}\right)\right. \\
& +g_{\tau \tau^{*}}^{(2 B)} K_{2+\tau \tau^{*}}^{(n)}\left(Q^{2}\right)-g_{\tau \tau^{*}}^{(3 B)} K_{1-\tau \tau^{*}}^{(n)}\left(Q^{2}\right)-g_{\tau \tau^{*}}^{(1 E)} K_{2-\tau \tau^{*}}^{(n)}\left(Q^{2}\right)+g_{\tau \tau^{*}}^{(4 E)} K_{1+\tau \tau^{*}}^{(n)}\left(Q^{2}\right) \\
& \left.+g_{\tau \tau^{*}}^{(2 C)} R_{2+\tau \tau^{*}}^{(n)}\left(Q^{2}\right)-g_{\tau \tau^{*}}^{(3 C)} R_{1-\tau \tau^{*}}^{(n)}\left(Q^{2}\right)-g_{\tau \tau^{*}}^{(1 F)} R_{2-\tau \tau^{*}}^{(n)}\left(Q^{2}\right)+g_{\tau \tau^{*}}^{(4 F)} R_{1+\tau \tau^{*}}^{(n)}\left(Q^{2}\right)\right],
\end{aligned}
$$

where $F_{i \pm \tau \tau^{*}}^{(n)}\left(Q^{2}\right)$ with $F=K, L, M, N, R$ are functions calculated in the soft-wall model. Functions $F_{i \pm \tau \tau^{*}}^{(n)}\left(Q^{2}\right)$ are written as

$$
\begin{aligned}
& F_{1 \pm \tau \tau^{*}}^{(n)}\left(Q^{2}\right)=\frac{1}{2}\left[F^{(n)}\left(Q^{2}, \tau^{*}, \tau\right) \pm F^{(n)}\left(Q^{2}, \tau^{*}+1, \tau+1\right)\right] \\
& F_{2 \pm \tau \tau^{*}}^{(n)}\left(Q^{2}\right)=\frac{1}{2}\left[F^{(n)}\left(Q^{2}, \tau^{*}, \tau+1\right) \pm F^{(n)}\left(Q^{2}, \tau^{*}+1, \tau\right)\right]
\end{aligned}
$$

and

$$
R_{i \pm \tau \tau^{*}}^{(n)}\left(Q^{2}\right)=\frac{1}{\kappa}\left[L_{i \pm \tau \tau^{*}}^{(n)}\left(Q^{2}\right)+N_{i \pm \tau \tau^{*}}^{(n)}\left(Q^{2}\right)\right]
$$



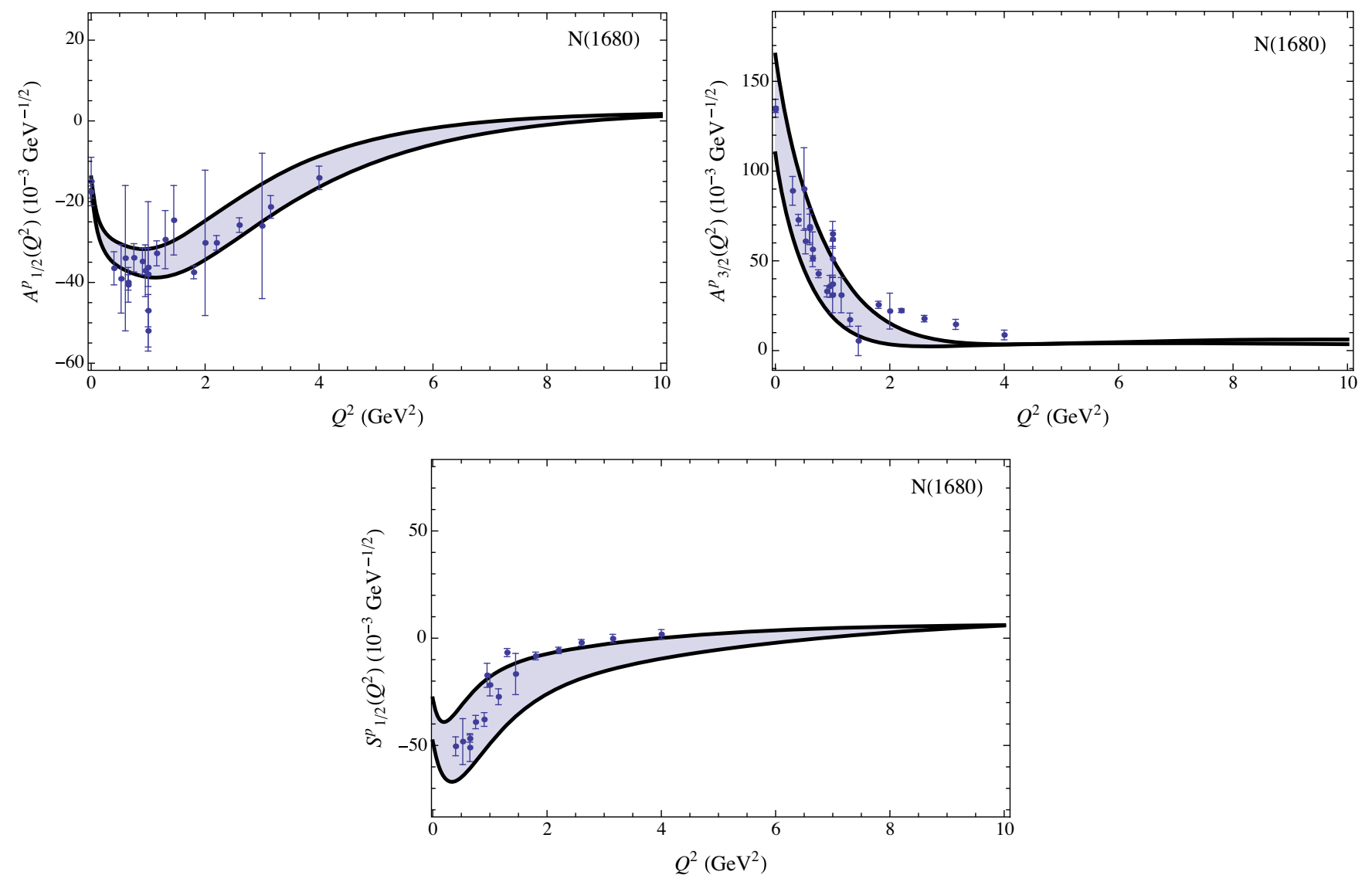

FIG. 10. Helicity amplitudes $A_{1 / 2}^{p}\left(Q^{2}\right)$ (left upper panel), $A_{3 / 2}^{p}\left(Q^{2}\right)$ (right upper panel), and $S_{1 / 2}^{p}\left(Q^{2}\right)$ (centered lower panel), for $N \gamma^{*} \rightarrow N(1680)$ transition up to $Q^{2}=10 \mathrm{GeV}^{2}$. Our results (shaded band) are compared with data taken from the CLAS Collaboration $[68,70,71]$, data analysis [81], and PDG [63].

For $n=0$ one gets

$$
\begin{aligned}
K^{(0)}\left(Q^{2}, \tau^{*}, \tau\right) & =\frac{\left(\tau^{*}-\frac{3}{2}\right) K\left(a, \tau^{*}, \tau\right)-K\left(a, \tau^{*}+2, \tau\right)}{2 \sqrt{\Gamma\left(\tau^{*}-1\right) \Gamma(\tau-1)}}, \\
L^{(0)}\left(Q^{2}, \tau^{*}, \tau\right) & =\frac{L\left(a, \tau^{*}, \tau\right)}{\sqrt{\Gamma\left(\tau^{*}-1\right) \Gamma(\tau-1)}}, \\
M^{(0)}\left(Q^{2}, \tau^{*}, \tau\right) & =\frac{M\left(a, \tau^{*}, \tau\right)}{2 \sqrt{\Gamma\left(\tau^{*}-1\right) \Gamma(\tau-1)}}, \\
N^{(0)}\left(Q^{2}, \tau^{*}, \tau\right) & =\frac{K\left(a, \tau^{*}, \tau\right)-2 K\left(a, \tau^{*}+2, \tau\right)}{2 \sqrt{\Gamma\left(\tau^{*}-1\right) \Gamma(\tau-1)}} .
\end{aligned}
$$

Here

$$
\begin{aligned}
& K\left(a, \tau^{*}, \tau\right)=\Gamma\left(\frac{\tau^{*}+\tau}{2}\right) B\left(a+1, \frac{\tau^{*}+\tau}{2}\right), \\
& L\left(a, \tau^{*}, \tau\right)=\Gamma\left(\frac{\tau^{*}+\tau+2}{2}\right) B\left(a+1, \frac{\tau^{*}+\tau}{2}\right), \\
& M\left(a, \tau^{*}, \tau\right)=\Gamma\left(\frac{\tau^{*}+\tau+1}{2}\right) B\left(a+1, \frac{\tau^{*}+\tau+1}{2}\right), \\
& N\left(a, \tau^{*}, \tau\right)=K\left(a, \tau^{*}, \tau\right)-2 K\left(a, \tau^{*}+2, \tau\right) .
\end{aligned}
$$

It is important to stress that at large values of $Q^{2}$ the form factors and helicity amplitudes for the electroexcitation of nucleon resonances are consistent with quark counting rules [54]. In particular, the sets of the form factors $h_{i}, G_{i},\left(G_{E}\right.$, $\left.G_{M}, G_{C}\right)$, and helicity amplitudes $\left(A_{1 / 2}, A_{3 / 2}, S_{1 / 2}\right)$ scale as

$$
\begin{aligned}
& h_{i}\left(Q^{2}\right) \sim \frac{1}{Q^{\tau+\tau^{*}-2}}, \quad G_{i}\left(Q^{2}\right) \sim \frac{1}{Q^{\tau+\tau^{*}}}, \\
& G_{E}\left(Q^{2}\right), G_{M}\left(Q^{2}\right), G_{C}\left(Q^{2}\right) \sim \frac{1}{Q^{\tau+\tau^{*}-2}}, \\
& A_{1 / 2}^{l}\left(Q^{2}\right), A_{3 / 2}^{l}\left(Q^{2}\right) \sim \frac{1}{Q^{\tau+\tau^{*}-1-2 l}}, \\
& S_{1 / 2}^{l}\left(Q^{2}\right) \sim \frac{1}{Q^{\tau+\tau^{*}-3-2 l}} .
\end{aligned}
$$

Model parameters (central values) used for each $\gamma^{*} N \rightarrow N^{*}$ transition are shown in Tables II and III. As in previous calculations we include the contributions of three leading twists. Also, to reduce a number of free parameters we drop the contribution to the form factors induced by the couplings $g_{\tau \tau^{*}}^{3 M}$ and $g_{\tau \tau^{*}}^{4 M}$.

Our results for the $Q^{2}$ dependence of the helicity amplitudes in the $\gamma^{*} N \rightarrow N^{*}$ transitions including a 

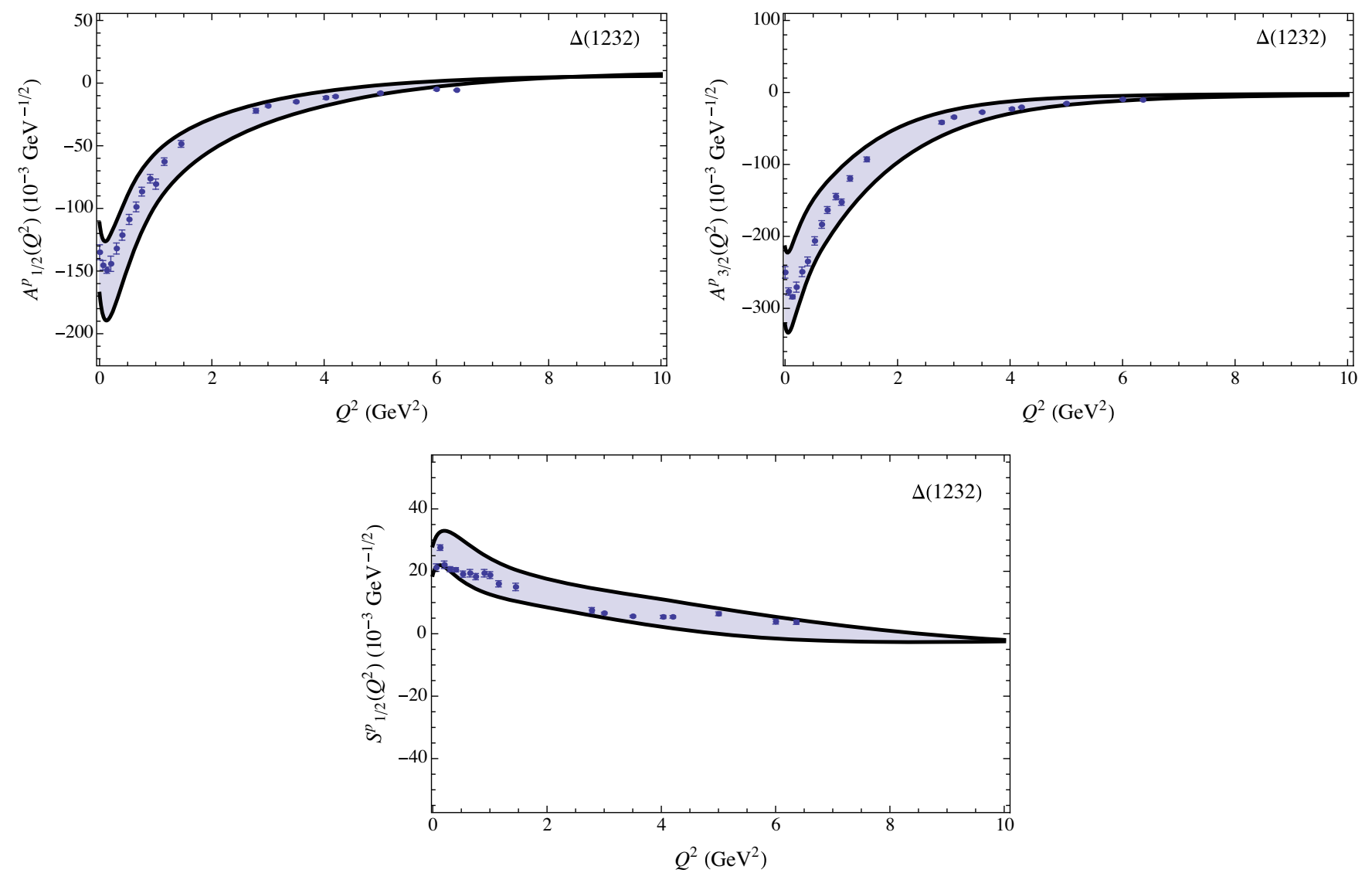

FIG. 11. Helicity amplitudes $A_{1 / 2}^{p}\left(Q^{2}\right)$ (left upper panel), $A_{3 / 2}^{p}\left(Q^{2}\right)$ (right upper panel), and $S_{1 / 2}^{p}\left(Q^{2}\right)$ (centered lower panel), for $N \gamma^{*} \rightarrow \Delta(1232)$ transition up to $Q^{2}=10 \mathrm{GeV}^{2}$. Our results (shaded band) are compared with data taken from the CLAS Collaboration [4] and PDG [63].

variation of the parameters (up to 20\%) are fully displayed in Figs. 1-11. In Figs. 1-3 we present the results for the modes with nucleon resonances having spin- $\frac{1}{2}$, which were not considered by us before and in addition in Figs. 4-14 we display the results for the nucleon resonances with higher spins $-\frac{3}{2}$ and $-\frac{5}{2}$. We compare our results to the data from the CLAS Collaboration (JLab) [4-6,68-72], other experiments [73-80], and world data analyses [81-83]. Also we consider in detail the observables of the $\gamma^{*} N \rightarrow$ $\Delta(1232)$ transitions: helicity amplitudes (Fig. 11), the $Q^{2}$ dependence of the magnetic form factor $G_{M}^{*}\left(Q^{2}\right)$ divided by the dipole form factor $3 D\left(Q^{2}\right)$ (Fig. 12), where $D\left(Q^{2}\right)=1 /\left(1+Q^{2} / 0.71 \mathrm{GeV}^{2}\right)^{2}$, the $Q^{2}$ dependence of the $R_{\mathrm{EM}}=E / M$ and $R_{\mathrm{SM}}=S / M$ ratios (Fig. 13 up to $5 \mathrm{GeV}^{2}$ and Fig. 14 up to $10 \mathrm{GeV}^{2}$ ), and magnetic dipole $\mu_{N \Delta}$ and electric quadrupole $Q_{N \Delta}$ moments:

$$
\begin{aligned}
& R_{\mathrm{EM}}\left(Q^{2}\right)=\frac{A_{1 / 2}\left(Q^{2}\right)-A_{3 / 2}\left(Q^{2}\right) / \sqrt{3}}{A_{1 / 2}\left(Q^{2}\right)+A_{3 / 2}\left(Q^{2}\right) \sqrt{3}}, \\
& R_{\mathrm{SM}}\left(Q^{2}\right)=\frac{S_{1 / 2}\left(Q^{2}\right) \sqrt{2}}{A_{1 / 2}\left(Q^{2}\right)+A_{3 / 2}\left(Q^{2}\right) \sqrt{3}},
\end{aligned}
$$

$$
\begin{aligned}
\mu_{N \Delta} & =\sqrt{\frac{M_{\Delta}}{M_{N}}} G_{M}^{*}(0), \\
Q_{N \Delta} & =-\frac{6}{M_{N} E_{N}} \sqrt{\frac{M_{\Delta}}{M_{N}}} G_{E}^{*}(0) .
\end{aligned}
$$

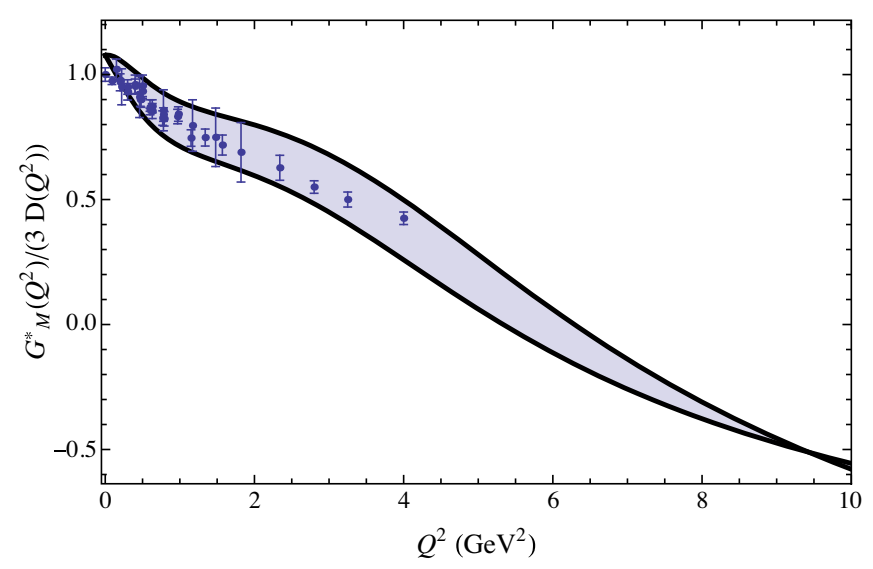

FIG. 12. $Q^{2}$ dependence of the magnetic form factor $G_{M}^{*}\left(Q^{2}\right)$ divided by the dipole form factor $3 D\left(Q^{2}\right)$ up to $Q^{2}=10 \mathrm{GeV}^{2}$. Our results are compared with existing data taken from [73$76,82,83]$. 

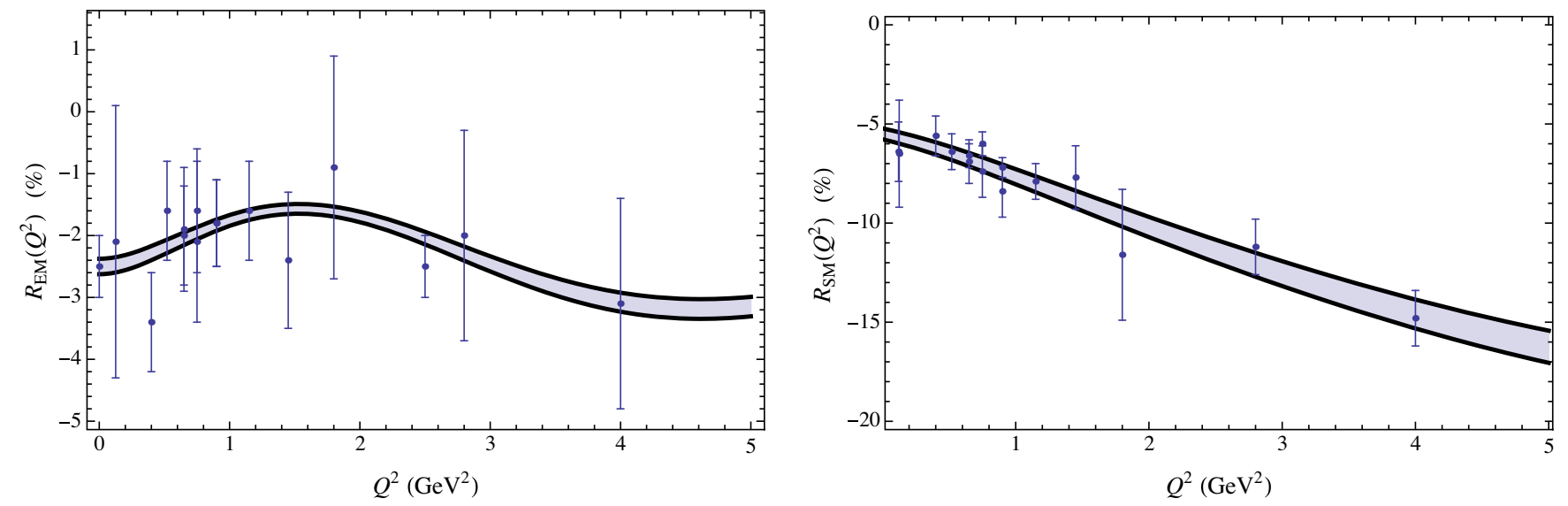

FIG. 13. $Q^{2}$ dependence of the ratios $R_{\mathrm{EM}}\left(Q^{2}\right)$ (left panel) and $R_{\mathrm{SM}}\left(Q^{2}\right)$ (right panel) up to $5 \mathrm{GeV}^{2}$. Our results are compared with data taken from PDG [63] and Refs. [77-79].
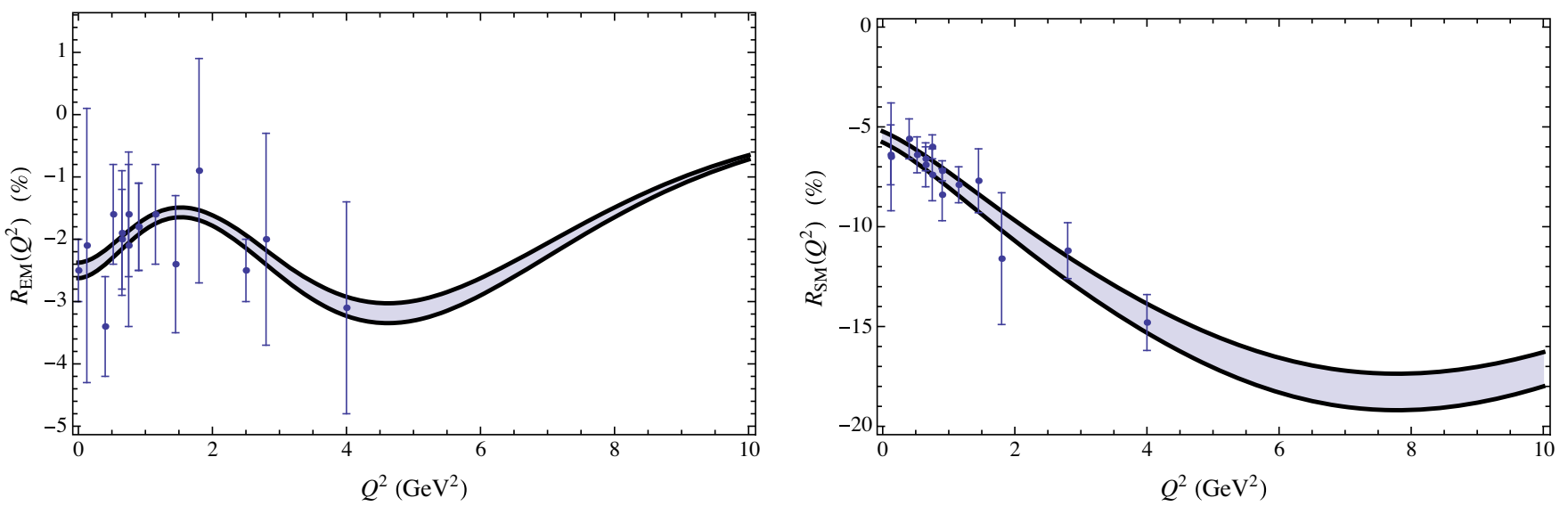

FIG. 14. $Q^{2}$ dependence of the ratios $R_{\mathrm{EM}}\left(Q^{2}\right)$ (left panel) and $R_{\mathrm{SM}}\left(Q^{2}\right)$ (right panel) up to $10 \mathrm{GeV}^{2}$. Our results are compared with data taken from PDG [63] and Refs. [77-79].

Note the magnetic $G_{M}^{*}\left(Q^{2}\right)$ and electric $G_{E}^{*}\left(Q^{2}\right)$ form factors are normalized as [82]

$G_{M}^{*}\left(Q^{2}\right)=G_{M}\left(Q^{2}\right) \frac{c_{\Delta}}{F_{1}^{+}}=-c_{\Delta}\left(A_{1 / 2}\left(Q^{2}\right)+A_{3 / 2}\left(Q^{2}\right) \sqrt{3}\right)$,

$G_{E}^{*}\left(Q^{2}\right)=G_{E}\left(Q^{2}\right) \frac{c_{\Delta}}{F_{1}^{+}}=c_{\Delta}\left(A_{1 / 2}\left(Q^{2}\right)-\frac{A_{3 / 2}\left(Q^{2}\right)}{\sqrt{3}}\right)$,

where

$$
c_{\Delta}=\frac{M_{N}}{|\mathbf{p}|} \sqrt{\frac{M_{N} E_{N}}{4 \pi \alpha M_{\Delta}}} .
$$

In Table IV our results for $R_{\mathrm{EM}}(0)$ are compared with existing data (PDG [63], MAMI experiment [77], LEGS Collaboration [80]) and some theoretical approaches [model based on partial-wave analysis (SAID) [84], approach based on dispersion relations and unitarity (DR) [85], and a relativistic quark model (RQM) [22] ].

For $R_{\mathrm{SM}}(0)$ we get $-5.5 \pm 0.5$. Our predictions for the moments $\mu_{N \Delta}$ and $Q_{N \Delta}$,

$\mu_{N \Delta}=3.7 \pm 0.4, \quad Q_{N \Delta}=-(0.09 \pm 0.01) \mathrm{fm}^{2}$

are in good agreement with the data (LEGS Collaboration [80])

TABLE IV. $R_{\mathrm{EM}}(0)$ ratio in (\%).

\begin{tabular}{lc}
\hline$-2.5 \pm 0.5$ & PDG [63] \\
$-2.5 \pm 0.1 \pm 0.2$ & MAMI [77] \\
$-3.07 \pm 0.26 \pm 0.24$ & LEGS [80] \\
$-2.0 \pm 0.2$ & SAID [84] \\
$-2.54 \pm 0.10$ & DR [85] \\
$-3.02 \pm 0.08$ & RQM [22] \\
$-2.5 \pm 0.5$ & (Our results) \\
\hline
\end{tabular}




$$
\begin{aligned}
& \mu_{N \Delta}=3.642 \pm 0.019 \pm 0.085 \\
& Q_{N \Delta}=-(0.108 \pm 0.009 \pm 0.034) \mathrm{fm}^{2}
\end{aligned}
$$

with the Mainz multipole analysis [82]:

$\mu_{N \Delta}=3.46 \pm 0.03, \quad Q_{N \Delta}=-(0.0846 \pm 0.0033) \mathrm{fm}^{2}$.

\section{SUMMARY}

We extended our formalism based on a soft-wall AdS/ QCD approach to the description of the electrocouplings of nucleons with nucleon resonances with high spins. All form factors and helicity amplitudes characterizing the electromagnetic transitions between nucleons and nucleon resonances are consistent with quark counting rules [54]. We fix free parameters in our approach using data from the CLAS Collaboration [4-6,68-72] and a compilation of the world analyses of the $N \pi$ electroproduction data [81]. In our calculations we adopt a variation of free parameters up to $20 \%$. The main success of our approach is based on analytical implementation of quark counting rules [54]. In the future we plan to further develop our approach for studying the thermal properties of nucleon resonances following our paper [52] and gluon content in hadrons. In particular, the study of gluon distributions in hadrons is important for shedding light on the nature of hadron hybrids-bound states of quarks and gluons, which are now under intensive study by the GlueX and CLAS Collaborations at JLab.

\section{ACKNOWLEDGMENTS}

This work was funded by "Verbundprojekt 05P2018Ausbau von ALICE am LHC: Jets und partonische Struktur von Kernen" (Förderkennzeichen No. 05P18VTCA1), "Verbundprojekt 05A2017-CRESST-XENON: Direkte Suche nach Dunkler Materie mit XENON1T/nT und CRESST-III. Teilprojekt 1" (Förderkennzeichen No. 05A17VTA)," by Agencia Nacional de Investigación y Desarrollo (Chile) under Grant No. 7912010025, by ANID PIA/APOYO AFB180002, by FONDECYT (Chile) under Grants No. 1191103 and No. 1180232, by the Tomsk State University Competitiveness Enhancement Program "Research of Modern Problems of Quantum Field Theory and Condensed Matter Physics," and Tomsk Polytechnic University Competitiveness Enhancement Program (Russia).

\section{APPENDIX: AdS/QCD ACTION FOR DESCRIPTION OF THE $\gamma+\frac{1}{2}^{+} \rightarrow \frac{1}{2}^{ \pm}$ TRANSITIONS}

The AdS/QCD action for description of the $\gamma+\frac{1}{2}^{+} \rightarrow \frac{1}{2}^{ \pm}$ transitions contains a free part $S_{0}$ describing the confined dynamics of AdS fields, and interaction part $S_{\text {int }}$ describing interactions of fermions with vector field with

$$
\begin{aligned}
S= & S_{0}+S_{\text {int }}, \\
S_{0}= & \int d^{4} x d z \sqrt{g} e^{-\varphi(z)}\left\{\mathcal{L}_{N}(x, z)+\mathcal{L}_{N^{*}}(x, z)+\mathcal{L}_{V}(x, z)\right\}, \\
S_{\text {int }}= & \int d^{4} x d z \sqrt{g} e^{-\varphi(z)}\left\{\mathcal{L}_{V N N}(x, z)+\mathcal{L}_{V N^{*} N}(x, z)\right. \\
& \left.+\mathcal{L}_{V N^{*} N^{*}}(x, z)\right\},
\end{aligned}
$$

where $\mathcal{L}_{N}, \mathcal{L}_{N^{*}}, \mathcal{L}_{V}(x, z)$ and $\mathcal{L}_{V N N}(x, z), \mathcal{L}_{V N^{*} N}(x, z)$, $\mathcal{L}_{V N^{*} N^{*}}(x, z)$ are the free and interaction Lagrangians, respectively. See details in Refs. [51-53]; term $\mathcal{L}_{V}(x, z)$ is specified in Eq. (3). Below we specify the interaction Lagrangian $\mathcal{L}_{V N^{*} N}(x, z)$, relevant for the $\gamma+\frac{1}{2}^{+} \rightarrow \frac{1}{2}^{ \pm}$ transitions. In particular,

$$
\mathcal{L}_{V N^{*} N}(x, z)=\sum_{i=+,-; \tau \tau^{*}} \bar{\psi}_{i, \tau^{*}}^{N^{*}}(x, z) \hat{\mathcal{V}}_{i, \tau \tau^{*}}^{N^{*} N}(x, z) \psi_{i, \tau}^{N}(x, z)+\text { H.c. },
$$

where

$$
\begin{aligned}
\hat{\mathcal{V}}_{ \pm, \tau \tau^{*}}^{N^{*} N}(x, z)= & c_{\tau \tau^{*}} Q \Gamma^{M} V_{M}(x, z) \\
& +d_{\tau \tau^{*}}\left[ \pm \frac{i}{4} \eta\left[\Gamma^{M}, \Gamma^{N}\right] V_{M N}(x, z)\right. \\
& \pm \frac{i}{4} \lambda z^{2}\left[\Gamma^{M}, \Gamma^{N}\right] \partial^{K} \partial_{K} V_{M N}(x, z) \\
& \pm g \Gamma^{M} i \Gamma^{z} V_{M}(x, z)+\zeta z \Gamma^{M} \partial^{N} V_{M N}(x, z) \\
& \left. \pm \xi z \Gamma^{M} i \Gamma^{z} \partial^{N} V_{M N}(x, z)\right] .
\end{aligned}
$$

Here $c_{\tau \tau^{*}}, d_{\tau \tau^{*}}, \eta, \lambda, g, \zeta$, and $\xi$ are the couplings fixed from description of the data on the $Q^{2}$ dependence of the $\gamma+$ $\frac{1}{2}^{+} \rightarrow \frac{1}{2}^{ \pm}$transitions. In the case of the $\gamma+\frac{1}{2}^{+} \rightarrow \frac{1}{2}^{+}$ transitions we use $c_{\tau \tau^{*}} \equiv d_{\tau \tau^{*}}$. 
[1] R. C. E. Devenish, T. S. Eisenschitz, and J. G. Korner, Phys. Rev. D 14, 3063 (1976); H. F. Jones and M. D. Scadron, Ann. Phys. (N.Y.) 81, 1 (1973).

[2] S. Capstick and B. D. Keister, Phys. Rev. D 51, 3598 (1995).

[3] I. G. Aznauryan and V. D. Burkert, Prog. Part. Nucl. Phys. 67, 1 (2012).

[4] I. G. Aznauryan et al. (CLAS Collaboration), Phys. Rev. C 80, 055203 (2009).

[5] I. G. Aznauryan and V. D. Burkert, Phys. Rev. C 85, 055202 (2012).

[6] V. I. Mokeev et al., Phys. Rev. C 93, 025206 (2016).

[7] S. Stajner, P. Achenbach, T. Beranek, J. Bericic, J. C. Bernauer, D. Bosnar, R. Bohm, L. Correa, A. Denig et al., Phys. Rev. Lett. 119, 022001 (2017).

[8] L. Tiator, D. Drechsel, S. S. Kamalov, and M. Vanderhaeghen, Eur. Phys. J. Special Topics 198, 141 (2011).

[9] D. Drechsel, S. S. Kamalov, and L. Tiator, Eur. Phys. J. A 34, 69 (2007).

[10] L. Tiator and M. Vanderhaeghen, Phys. Lett. B 672, 344 (2009).

[11] R. A. Arndt, W. J. Briscoe, M. W. Paris, I. I. Strakovsky, and R. L. Workman, Chin. Phys. C 33, 1063 (2009).

[12] A. V. Anisovich, V. Burkert, E. Klempt, V. A. Nikonov, A. V. Sarantsev, and U. Thoma, Eur. Phys. J. A 49, 67 (2013).

[13] I. G. Aznauryan, V. D. Burkert, H. Egiyan, K. Joo, R. Minehart, and L. C. Smith, Phys. Rev. C 71, 015201 (2005).

[14] G. V. Efimov, M. A. Ivanov, and V. E. Lyubovitskij, Few Body Syst. 6, 17 (1989); Acta Phys. Austriaca 6, 17 (1989).

[15] K. Bermuth, D. Drechsel, L. Tiator, and J. B. Seaborn, Phys. Rev. D 37, 89 (1988).

[16] W. Konen and H. J. Weber, Phys. Rev. D 41, 2201 (1990); R. H. Stanley and H. J. Weber, Phys. Rev. C 52, 435 (1995).

[17] F. Stancu and P. Stassart, Phys. Rev. D 41, 916 (1990).

[18] Z. P. Li, V. Burkert, and Z. J. Li, Phys. Rev. D 46, 70 (1992).

[19] R. W. Lourie, Z. Phys. C 50, 345 (1991).

[20] F. Cardarelli, E. Pace, G. Salme, and S. Simula, Phys. Lett. B 397, 13 (1997).

[21] Y. B. Dong, K. Shimizu, A. Faessler, and A. J. Buchmann, Phys. Rev. C 60, 035203 (1999).

[22] A. Faessler, T. Gutsche, B. R. Holstein, V. E. Lyubovitskij, D. Nicmorus, and K. Pumsa-ard, Phys. Rev. D 74, 074010 (2006).

[23] I. G. Aznauryan, Phys. Rev. C 76, 025212 (2007).

[24] D. Y. Chen and Y. B. Dong, Commun. Theor. Phys. 50, 142 (2008).

[25] G. Ramalho and K. Tsushima, Phys. Rev. D 81, 074020 (2010).

[26] G. Ramalho and K. Tsushima, Phys. Rev. D 82, 073007 (2010).

[27] G. Ramalho and M. T. Pena, Phys. Rev. D 84, 033007 (2011).

[28] S. Parsaei and A. Akbar Rajabi, Eur. Phys. J. Plus 132, 413 (2017).

[29] M. A. Ivanov, G. Nurbakova, and Z. Tyulemissov, Phys. Part. Nucl. Lett. 15, 1 (2018).

[30] D. Jido, M. Döring, and E. Oset, Phys. Rev. C 77, 065207 (2008).

[31] T. Bauer, J. Gegelia, and S. Scherer, Phys. Lett. B 715, 234 (2012); T. Bauer, S. Scherer, and L. Tiator, Phys. Rev. C 90, 015201 (2014).
[32] M. Hilt, T. Bauer, S. Scherer, and L. Tiator, Phys. Rev. C 97, 035205 (2018).

[33] V. M. Braun et al., Phys. Rev. Lett. 103, 072001 (2009); I. V. Anikin, V. M. Braun, and N. Offen, Phys. Rev. D 92 , 014018 (2015).

[34] T. M. Aliev and M. Savci, Phys. Rev. D 88, 056021 (2013); T. M. Aliev, T. Barakat, and K. Simsek, Phys. Rev. D 100, 054030 (2019).

[35] I. T. Obukhovsky, A. Faessler, D. K. Fedorov, T. Gutsche, and V. E. Lyubovitskij, Phys. Rev. D 84, 014004 (2011).

[36] I. T. Obukhovsky, A. Faessler, T. Gutsche, and V.E. Lyubovitskij, Phys. Rev. D 89, 014032 (2014); I. T. Obukhovsky, A. Faessler, D. K. Federov, T. Gutsche, and V. E. Lyubovitskij, Proc. Sci. Baldin, ISHEPP-XXI2012 (2012) 061.

[37] I. T. Obukhovsky, A. Faessler, D. K. Fedorov, T. Gutsche, and V. E. Lyubovitskij, Phys. Rev. D 100, 094013 (2019).

[38] G. Eichmann, Phys. Rev. D 84, 014014 (2011).

[39] D. J. Wilson, I. C. Cloet, L. Chang, and C. D. Roberts, Phys. Rev. C 85, 025205 (2012).

[40] J. Segovia, B. El-Bennich, E. Rojas, I. C. Cloet, C. D. Roberts, S.S. Xu, and H. S. Zong, Phys. Rev. Lett. 115, 171801 (2015).

[41] G. Eichmann, H. Sanchis-Alepuz, R. Williams, R. Alkofer, and C. S. Fischer, Prog. Part. Nucl. Phys. 91, 1 (2016).

[42] Y. Lu, C. Chen, Z. F. Cui, C. D. Roberts, S. M. Schmidt, J. Segovia, and H. S. Zong, Phys. Rev. D 100, 034001 (2019).

[43] C. Chen, Y. Lu, D. Binosi, C. D. Roberts, J. RodríguezQuintero, and J. Segovia, Phys. Rev. D 99, 034013 (2019).

[44] C. S. An and B.S. Zou, Eur. Phys. J. A 39, 195 (2009); Chin. Phys. C 34, 245 (2010).

[45] G. Ramalho and K. Tsushima, Phys. Rev. D 84, R051301 (2011); G. Ramalho, Phys. Lett. B 759, 126 (2016); Phys. Rev. D 95, 054008 (2017).

[46] H. R. Grigoryan, T.-S. H. Lee, and H. U. Yee, Phys. Rev. D 80, 055006 (2009).

[47] G. F. de Teramond and S. J. Brodsky, AIP Conf. Proc. 1432, 168 (2012).

[48] T. Gutsche, V. E. Lyubovitskij, I. Schmidt, and A. Vega, Phys. Rev. D 87, 016017 (2013).

[49] G. Ramalho and D. Melnikov, Phys. Rev. D 97, 034037 (2018).

[50] G. Ramalho, Phys. Rev. D 96, 054021 (2017).

[51] T. Gutsche, V. E. Lyubovitskij, and I. Schmidt, Phys. Rev. D 97, 054011 (2018).

[52] T. Gutsche, V. E. Lyubovitskij, and I. Schmidt, Nucl. Phys. B952, 114934 (2020).

[53] T. Gutsche, V. E. Lyubovitskij, and I. Schmidt, Phys. Rev. D 101, 034026 (2020).

[54] S. J. Brodsky and G. R. Farrar, Phys. Rev. Lett. 31, 1153 (1973); V. A. Matveev, R. M. Muradian, and A. N. Tavkhelidze, Lett. Nuovo Cimento 7, 719 (1973).

[55] V. E. Lyubovitskij and I. Schmidt, Phys. Rev. D 102, 034011 (2020).

[56] T. Gutsche, V. E. Lyubovitskij, I. Schmidt, and A. Vega, Phys. Rev. D 85, 076003 (2012); A. Vega, I. Schmidt, T. Gutsche, and V. E. Lyubovitskij, Phys. Rev. D 83, 036001 (2011).

[57] T. Branz, T. Gutsche, V. E. Lyubovitskij, I. Schmidt, and A. Vega, Phys. Rev. D 82, 074022 (2010); A. Vega, I. Schmidt, 
T. Branz, T. Gutsche, and V. E. Lyubovitskij, Phys. Rev. D 80, 055014 (2009).

[58] T. Gutsche, V. E. Lyubovitskij, I. Schmidt, and A. Vega, Phys. Rev. D 86, 036007 (2012).

[59] T. Gutsche, V. E. Lyubovitskij, I. Schmidt, and A. Vega, Phys. Rev. D 87, 056001 (2013).

[60] T. Gutsche, V. E. Lyubovitskij, and I. Schmidt, Phys. Rev. D 94, 116006 (2016); T. Gutsche, V. E. Lyubovitskij, I. Schmidt, and A. Vega, Phys. Rev. D 91, 114001 (2015).

[61] T. Gutsche, V. E. Lyubovitskij, and I. Schmidt, Phys. Rev. D 96, 034030 (2017).

[62] T. Gutsche, V. E. Lyubovitskij, I. Schmidt, and A. Y. Trifonov, Phys. Rev. D 99, 054030 (2019); 99, 114023 (2019).

[63] P. A. Zyla et al. (Particle Data Group), Prog. Theor. Exp. Phys. 2020, 083C01 (2020).

[64] V. I. Mokeev et al., Phys. Lett. B 805, 135457 (2020).

[65] H. R. Grigoryan and A. V. Radyushkin, Phys. Rev. D 76, 095007 (2007).

[66] A. Kadeer, J. G. Körner, and U. Moosbrugger, Eur. Phys. J. C 59, 27 (2009).

[67] A. Faessler, T. Gutsche, M. A. Ivanov, J. G. Körner, and V. E. Lyubovitskij, Phys. Rev. D 80, 034025 (2009); T. Branz, A. Faessler, T. Gutsche, M. A. Ivanov, J. G. Körner, V. E. Lyubovitskij, and B. Oexl, Phys. Rev. D 81, 114036 (2010); T. Gutsche, M. A. Ivanov, J. G. Körner, V. E. Lyubovitskij, V. V. Lyubushkin, and P. Santorelli, Phys. Rev. D 96, 013003 (2017).

[68] K. Park et al. (CLAS Collaboration), Phys. Rev. C 91, 045203 (2015).
[69] E. Golovatch et al. (CLAS Collaboration), Phys. Lett. B 788, 371 (2019).

[70] V. I. Mokeev and I. G. Aznauryan, Int. J. Mod. Phys. Conf. Ser. 26, 1460080 (2014).

[71] M. Dugger et al. (CLAS Collaboration), Phys. Rev. C 79, 065206 (2009).

[72] V. I. Mokeev et al. (CLAS Collaboration), Phys. Rev. C 86, 035203 (2012).

[73] W. Bartel, B. Dudelzak, H. Krehbiel, J. McElroy, U. MeyerBerkhout, W. Schmidt, V. Walther, and G. Weber, Phys. Lett. 28B, 148 (1968).

[74] S. Stein, W. B. Atwood, E. D. Bloom, R. L. A. Cottrell, H. DeStaebler, C. L. Jordan, H. G. Piel, C. Y. Prescott, R. Siemann, and R. E. Taylor, Phys. Rev. D 12, 1884 (1975).

[75] V. V. Frolov et al., Phys. Rev. Lett. 82, 45 (1999).

[76] F. Foster and G. Hughes, Rep. Prog. Phys. 46, 1445 (1983).

[77] R. Beck et al., Phys. Rev. Lett. 78, 606 (1997).

[78] T. Pospischil et al., Phys. Rev. Lett. 86, 2959 (2001).

[79] C. Mertz et al., Phys. Rev. Lett. 86, 2963 (2001).

[80] G. Blanpied et al., Phys. Rev. C 64, 025203 (2001).

[81] V. D. Burkert, R. De Vita, M. Battaglieri, M. Ripani, and V. Mokeev, Phys. Rev. C 67, 035204 (2003).

[82] L. Tiator, D. Drechsel, S. S. Kamalov, and S. N. Yang, Eur. Phys. J. A 17, 357 (2003).

[83] S. S. Kamalov, S. N. Yang, D. Drechsel, O. Hanstein, and L. Tiator, Phys. Rev. C 64, 032201 (2001).

[84] R. A. Arndt, W. J. Briscoe, I. I. Strakovsky, and R. L. Workman, Phys. Rev. C 66, 055213 (2002).

[85] O. Hanstein, D. Drechsel, and L. Tiator, Nucl. Phys. A632, 561 (1998). 